\title{
PULSE SYNCHRONIZATION SYSTEM (PSS)
}

WORK PERFORMED UNDER CONTRACT TO LAWRENCE LIVERMORE LABORATORIES
0.9660315

JUNE 1977

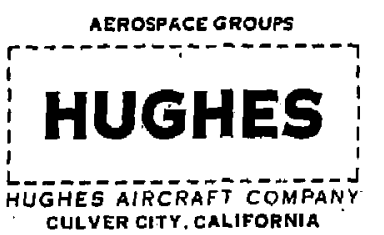




\section{PULSE SYNCHRONIZA'TION SYSTEM (PSS)}

Contract No. 9660315

Wiork Performed Under Contract to Lawrence Livermore Laboratories

June 1977

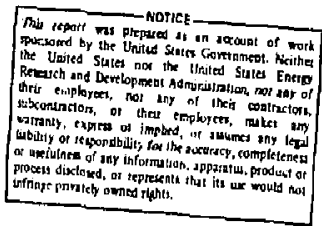

Electro-Optical and Data Systems Group

Lase: Division

AEROSPACE GROUPS

Hughes Aircraft Company * Culver City, California 
CONTENTS

1.0 INTRODUCTION AND SUMMARY $\ldots \ldots \ldots \ldots \ldots$

2.0 SYSTEM DESCRIPTION . . ................ 3

2.1 Description - Optical Layout (Figure 2-1) ........ 3

2.2 Pulse Synchronization System Description - Signal

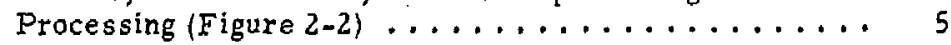

2.3 System Photographs ................. 7

3.0 SYSTEM ALIGNMENT AND OPERATION ............ 11

3.1 System Alignment ................... 11

3.2 System Operation ................... 16

4.0 PHYSICAL DESCRIPTION .................. 23

4.1 Description - Circuit Board Al (Figure 4-1) ........ 23

4. 2 Description - Circuit Board A2 (Figure 4-2) ....... 25

4.3 Description - Circuit Board A3 (Figure 4-3) . . . . . . 27

4.4 Description - Circuit Board A4 (Figure 4.4) ........ 29

4.5 Description - Circuit Board A5 (Figure 4-5) ....... 31

4-6 Description - Circuit Board A6 (Figure 4-6) ....... 33

4-7 Description - Circuit Board A7 (Figure 4-7) ....... 35

4.8 Description - Circuit Board A8 (Figure 4-8) . . . . . 37

4-9 Description - Circuit Board A9 (Figure $1-9$ ) ........ 39

4-10 Description - Blast Shutter and Intracavity Shutter
Operation (Figure $6-10) \ldots \ldots \ldots \ldots$. . . . . . .

4-11 Description - Chopper Reference Signal Circuit

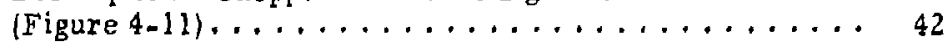

4-12 Description - Laser Hookup and Operation (Figure 4-12) . . 43

4-13 Description - Detectors and Preamplifier (Figure 4-13) .. 46

4-14 Electronics Control Panel (Figure 4-14) ........ 47 


\section{CONTENTS (Continued)}

5.0 SUPPORTING CALCULATIONS ................ 51

5.1 Characterization of the Signals ............ 51

5.2 The Carrier Signal to Noise Ratio $\left(\mathrm{SNR}_{\mathrm{c}}\right) \ldots \ldots \ldots . \ldots . . . . .54$

5.3 The Double LO Technique for Position Measurement..... 57

5.4 LO Beamsplitter Analysis ................. 61

5.5 Effects of Aberrations on SNR ............... 64

5.6 Ghost Signals ..................... 67

5.7 System Performance Estimate ............... 73

5.8 Appendix Calculation of Degradation in $S_{N R}$ Due to

Envelope Detection ................... 77 


\section{LIST OF ILLUSTRATIONS}

Figure

Page

1-1 Waveform Relationships............... 2

2-1 Optical Layout $\ldots \ldots \ldots \ldots \ldots \ldots \ldots \ldots \ldots \ldots \ldots \ldots$

2-2 Electronics - Signal Frocessing Diagram ........ 6

3-1 Dual Lo Alignment Detector Preamplifier Outputs for Detector Motions . . . . . . . . . . . . 17

$3-2 \quad$ IF signal waveforms $\ldots \ldots \ldots \ldots \ldots \ldots \ldots \ldots \ldots$

4-1 Electronics - Circuit Board AI - Shutter Control ..... 24

4-2 Electronics - Circuit Board A2 - Post Tuned Filter . . . . 26

4-3 Electronics - Circuit Board A3 - Boost Amplifier and Envelope Detector ................ 28

4-4 Electronics - Circuit Board A4-Pre-Filter. . . . . . . 30

4-5 Electronics - Circuit Board $A 5$ - Dither Oscillator . . . . 32

4-6 Electronics - Circuit Board A6-Dither Drive ... . . . 34

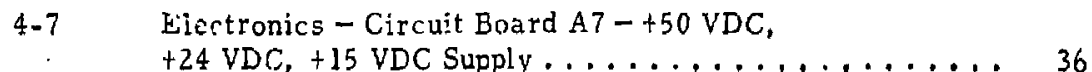

4-8 Elecironics - Circuit Board A8-Dual \pm 15 VDC

4-9 Electronics - Circuit Board A9 - Dual +24 VDC

4-10 Electronics - Optical Table - Shutter Systems Box .... 41

4-11 Electronics - Optical Table-Chopper Reference

Signal Circuit ...................... 42

4-12 Laser Hookup and Operation ................ 44

4-13 Electronics - Optical Table - Detectors and Preamplifier ..................... 46

4-14 Electronics Control Panel ... . . . . . . . . . . . . 49

5-1 Variation of Signal Current with Path Length Difference ..................... 58 


\section{ILLUSTRATIONS (Continued)}

Figure

Page

$5-2$ Dependence of SNR on the Ratio $\Delta / 2 \ldots \ldots \ldots \ldots$

5-3 $\quad$ LO Beamsplitter Configuration.............. 61

$5-4$ Geometry of a Specular Reflection ............ 68

$5,5 \quad$ Degradation Factor .................. 75

5-6 Estimated Position Error Versus Signal Path

Transmission ..................... 76 


\subsection{INTRODUCTION AND SUMMARY}

The following pages are intended to serve both as an operations manual, as well as a documentation of the backup analyses pertinent to the design as delivered. A history of earlier unsuccessful versions of the Pulse Synchronization System (PSS) is not included because of the lack of relevance to the present system and the delay that would be incurred in the delivery of this technical manual.

The function of the PSS is to synchronize the time of arrival at the fusion target of laser pulses that are propagated through the 20 amplifier chains of the SHIVA laser. The positional accuracy requirement is $\pm 1.5 \mathrm{mrn}( \pm 5 \mathrm{psec})$, and is obtained by the PSS with a wide margin factor. The principle of operation is illustrated in Figure 1-1. The PSS NdYAG laser injects a $\mathrm{CW}$ mode locked pulse train into the SHIVA oscillator chain. Nineteen of the amplifier chains are shuttered out, and the PSS pulse train is allowed to propagate down the selected amplifier chain to a reflective sphere placed at the focus of the last lens. The reflected beam returns to the PSS and is heterodyned with a frequency shifted portion of the laser output. The amplitude of the beat frequency current depends on the relative optical path lengths of the local oscillator (LO) path and the signal path. The method by which this behavior is exploited to obtain the required position accuracy is illustrated in Figure 1-1b. A chopper directs the LO beam alternately through two different LO optical path lengths: $\mathrm{LO}_{1}$ (the dashed response curve), and $\mathrm{LO}_{2}$ (the solid response curve). A phase lock amplifier with the chopper as a reference then detects the difference of these two curves yielding a meter reading that varies with the optical path difference $X$ as shown in Figure 1-1c. If $X$ is adjusted to the central null, then the 
a) LASER

OUTPUT
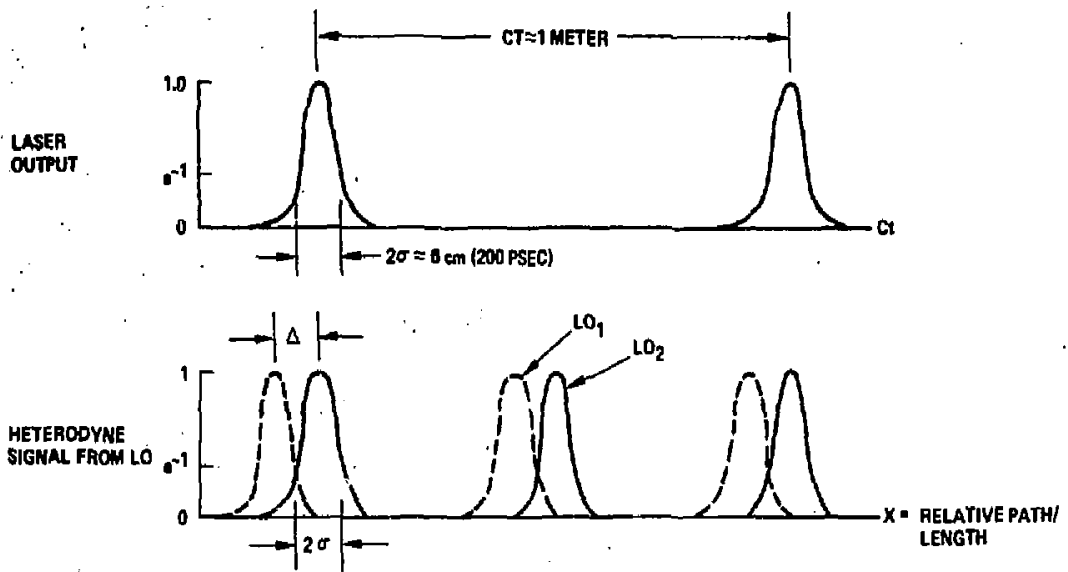

c) METER REAIDINE

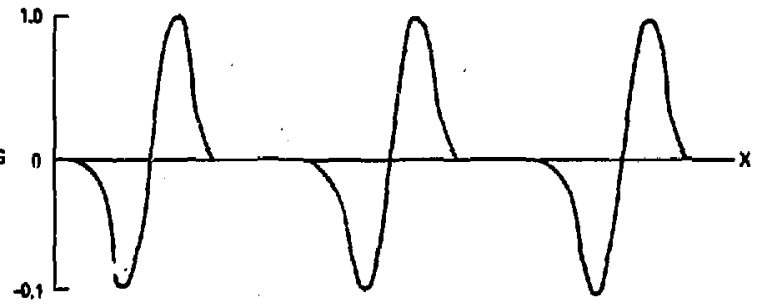

Figure 1-1. Waveform relationships.

meter will remain nulled as the signal strength varies; consequently, when each amplifier chain is successively switched in and its optical path length adjusted to give a meter null, the path lengths will be synchronized independent of variations in signal strength.

Theoretical performance estimates are summarized in Figure 5-5. These estimates are approximately verified when the PSS is tested by itself. When working into the CYCLOPS laser, the PSS was found $(6 / 17 / 77)$ to be disturbed by deep fading which appeared to be related to the time varying turbulent distortions observed on the PFC camera. The CYCLOPS attenuation was augmented to a total of $90 \mathrm{~dB}$ and the meter observed to go through a null upon translation of the LO cat's eye; however, the resolution was considerably worse then $\pm 1.5 \mathrm{~mm}$. A clarification of this noise mechanism may be obtained through experiments $(6 / 21 / 77)$ currently underway at Livermore. 


\subsection{SYSTEM DESCRIPTION}

\section{1 DESCRIPTION - OPTICAL LA YOUT (FIGURE 2-1)}

The modelocked output of the Quantronixs laser is monitored through the rear mirror. Although pulsewidths of less than 150 picosec are possible by careful alignment, a 250 picosec $1 /$ e width pulse will be utilized. This is necessary to minimize the drift in the luckin meter reading caused by spurious periods of relaxation oscillations of the laser. Greater stability and quiet operation are obtained by allowing the pulsewidth to grow. This stabilization has been effected by addition of a $1 \mathrm{~mm}$ etalon.

The beam is expanded to $4 \mathrm{~mm}$ and collimated by a $4 \mathrm{X}$ beam expander. The laser output, due to thermal birefringence, is not unipolar and a good polarizer is added externally. The beam passes through a half wave plate. Rotation of this plate allows control of the energy in the polarization selected by the polarizing beamsplitter to be the LO beam. The beam is split by the beamsplitter into the signal and local oscillator (LO) beams.

The signal beam traverses a quarterwave plate and exits circularly polarized. The laser chain polarization device selects one polarization. The attenuated return signal beam $(-90 \mathrm{~dB})$ passes again through this quarter waveplate and is circularly polarized. The polarizing beamsplitter puts a component into the detection arm. The beam is folded and enters a Wollaston prism inclined at 45 degrees relative to the signal polarization. The signal is thus divided and impinges on the two detectors.

The LO beam is folded upon leaving the beamsplitter. It then passes through the "dual LO oscillator device." The beam is equally split into two beams which are spatially separated. A chopping blade alternately selects one and then the other of these dual beams. In the figure, it is seen that the 


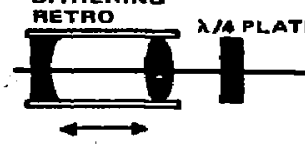

LUAL LO BEAM

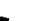
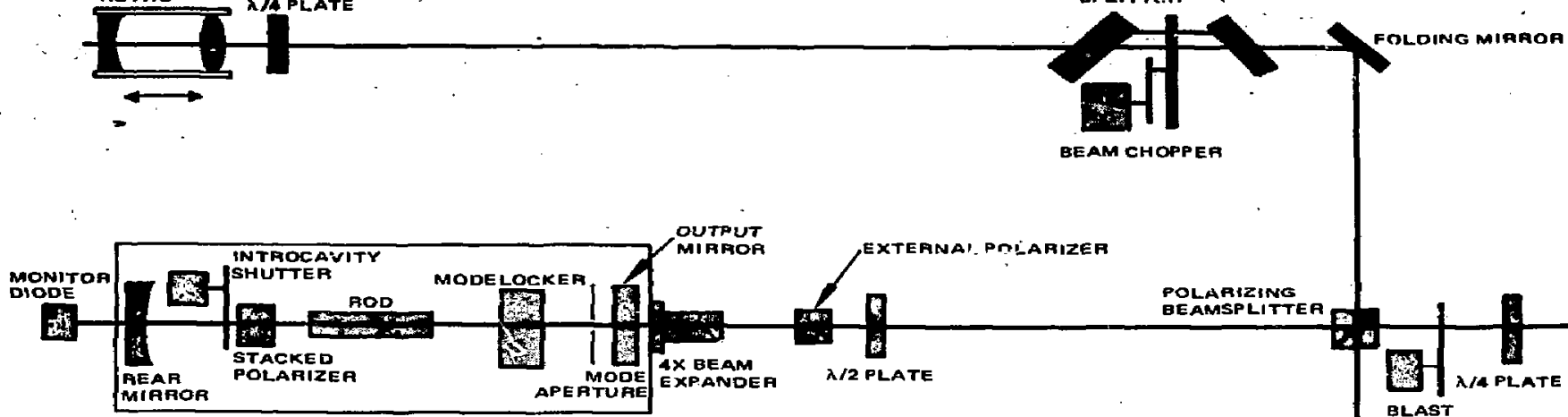

PollaRizing PolaAizing

BEAM CHOPPEA

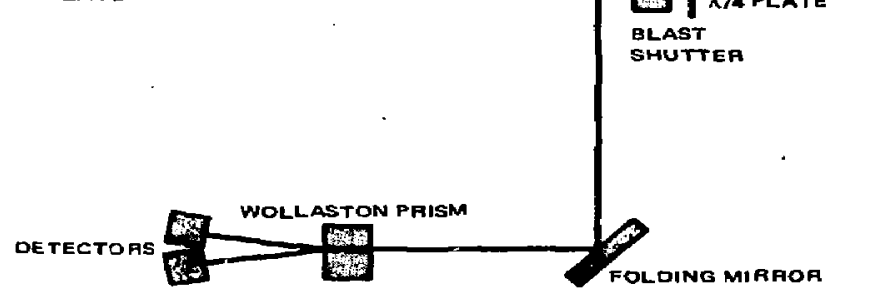

Figure 2-1. Optical layout. 
upper beam travels a farther distance while passing through the device. This provides the two LO beams with differing path lengths. The beams (if both were allowed to pass) recombine and enter the dithering retro. The combination of quarter waveplate and specular reflection off of the rear retro surface rotate the LO polarization by 90 degrees. The retro is translating back and forth along the optical axis. This phase modulation of the beam provides the heterodyne frequency offset. The beam is retro-reflected and shifted in frequency. It makes a second pass through the dual device doubling the path length difference. Since it is now rotated in polarization, it passes through the polarizing beamsplitter into the detection arm. It is polarized at right angles to the signal polarization and is split by the Wollaston and placed onto both detectors. The heterodyne beat is detected by both detectors. Due to the quadrature polarization the beat signals are out of phase by 180 degrees, and when differenced, add. Noise, or self beats of either the signal or local oscillat $r$ beams are in phase and when differenced are attenuated (Ideally canceled). The resulting $c$ fferenced signal then enters the electronics for signal processing.

\section{2 PULSE SYNCHRONIZATION SYSTEM DESCRIPTION - SIGNAL PROCESSING (FIGURE 2-2)}

A heterodyne signal between the local oscillator and signal returns is detected by both detectors. This detected heterodyine frequency offset signal is time varying. It is actually the detected phase modulation of the LO bearn imparted by the dither motion. Its amplitude depends on the relative path length difference between the LO and sigral optical paths. The balanced detection system reduces noise components, and chcpping transients. Since the LO beam can travel along two differing paths, a square wave amplitude modulation results on the detected heterodyne signnl. The modulation index depends on the relative path length difference between the median LO path and signal path. It approaches zero when the median path and signal path length difference is an integral number of laser resonator round trip cavity lengths. It is this null or zero that is detected by the lockin amplifier, that indicates path length equalization. 


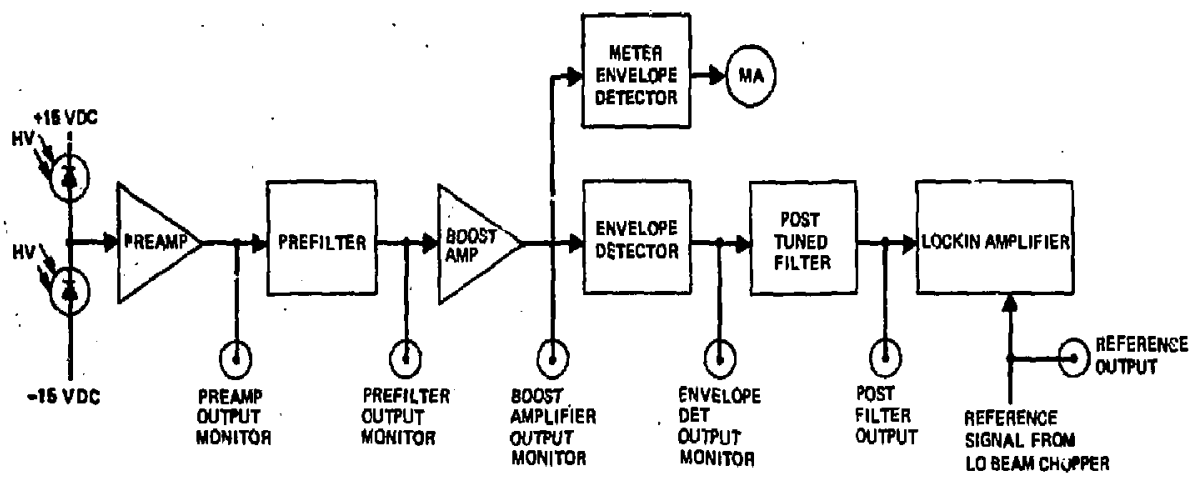

Figure 2-2. Electronic s - signal processing diagram.

The detected beat signa: is first current mode differenced (balanced detection) and then preamplified. Although a large LO noise rejection is achieved by the balanced detection, chopping noise transients are $\sim 80 \mathrm{~dB}$ above the detected signal. A prefilter is added and in conjunction with the - balanced detection gives rejection providing the necessary attenuation of these transients.

The prefilter is required for two reasons; 1) the following large amplification stages would be saturated by the large chopping waveiorms, and 2) the envelope of the carrier becomes ambiguous at the dither mirror turnaround and must be attenuated. . The prefilter employs 4 stages of $3 \mathrm{rd}$ order highpass filters. A small price is paid since the heterodyne signal is varying from near zero frequency to $100 \mathrm{kHz}$. This highpass filter causes a modulation of the detected waveform at twice the dither rate. Since the LO chop frequancy is significantly higher, the result is only a loss in average signal as recorded by the lockin amplifier.

Following the prefilter, the signal level is boosted to 1 volts peak to peak for use in a diode envelope detector. Two amplifiers with switch settable gains of one (1) and ten (10) and one hundred (100) are followed by a 
continuougly variable stage of one (1) to one hundred (100). A boost in signal level of one (1) to five hundred thousand $(500,000)$ is possible.

the signal is split and sent to two buifered envelope detectors. A large time constant on the meter detector provides a dc output to drive a signal level meter. This is calibrated to indicate the required 1 volt $p-p$ signal. The second, shorter time constant detector demodulates the square wave $A M$ modulation.

In order to increase the dynamic reserve of the lockin amplifier, a post tuned filter centered on the chopped LO frequency is utilized. This is a variable $Q$, variable frequency filter. It is set with the chopped frequency at center and a $Q$ of 5-10. The signal is detected by the lockin amplifier providing a meter indication. A meter null indicates equalization (integral number of laser cavity lengths $L$ ).

\subsection{SYSTEM PHOTOGRAPHS}

The photographs included below serve to illustrate the delivered hardware, which includes:

1. Laser head and optical table

2. Laser power supply

3. Laser mode locker

4. Spectra physics detector and power supply

5. Spare optical and electronic parts (not shown)

The digital counter and the oscilloscope were not deliverable items, 

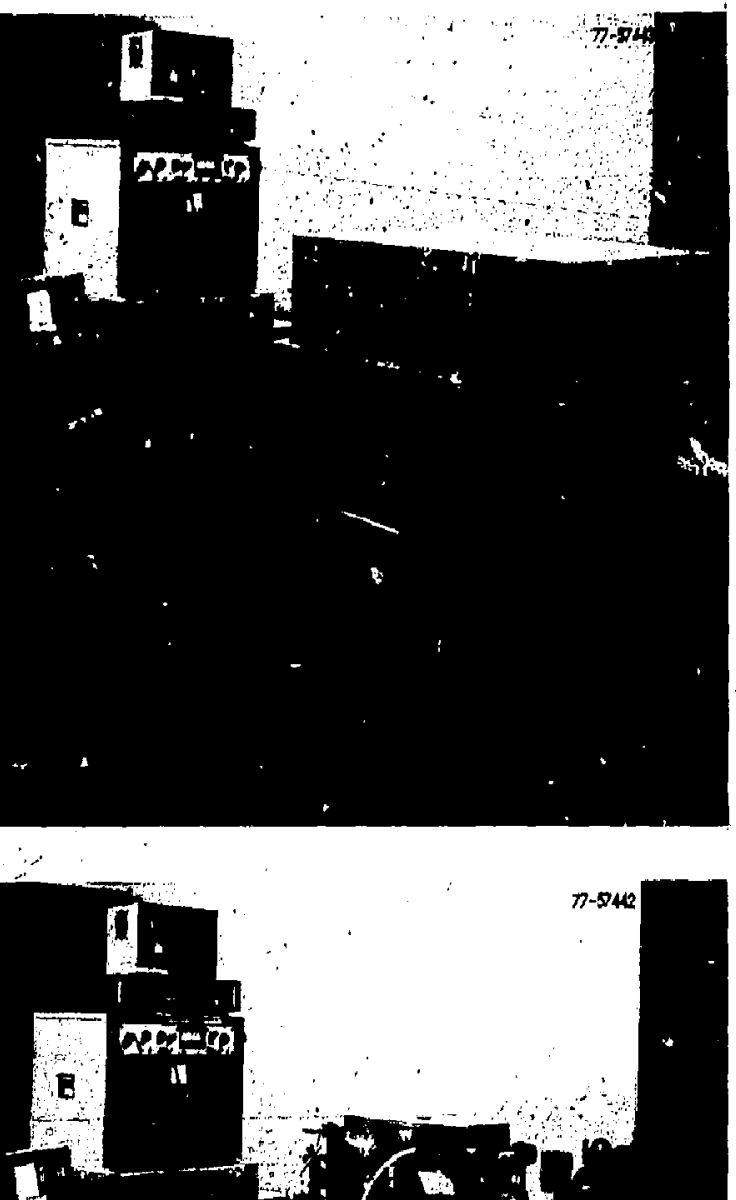

I

I

I

I

I

I

$+$

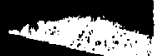

I

I

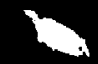




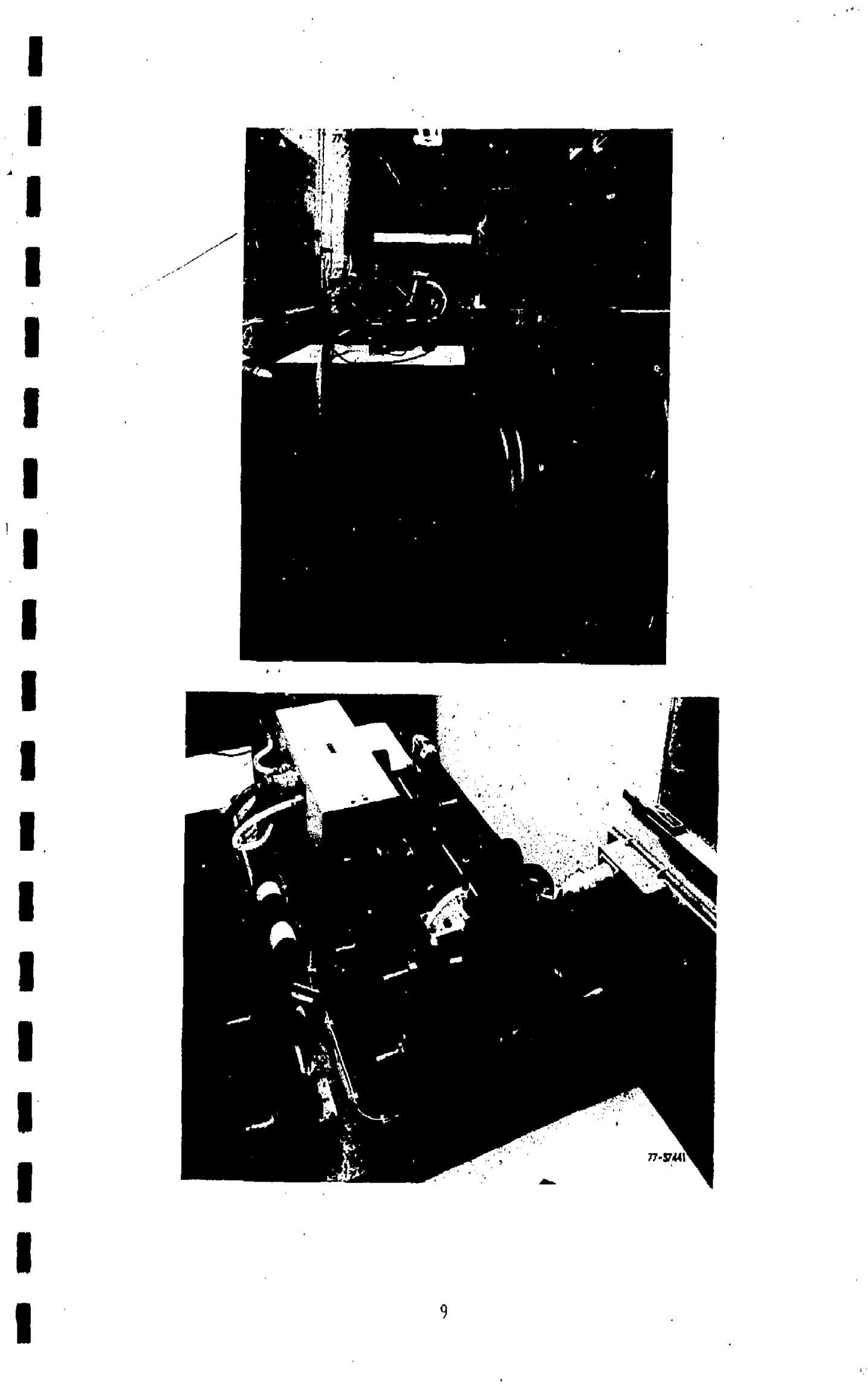




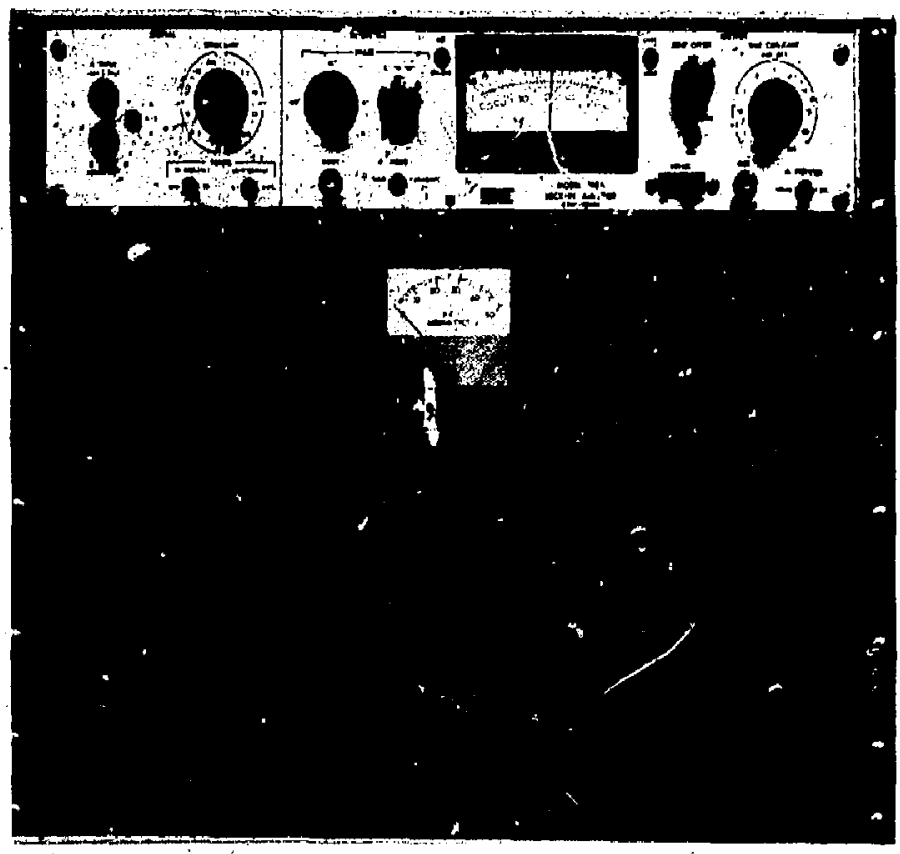

I

I

I

I

I

I

I

I

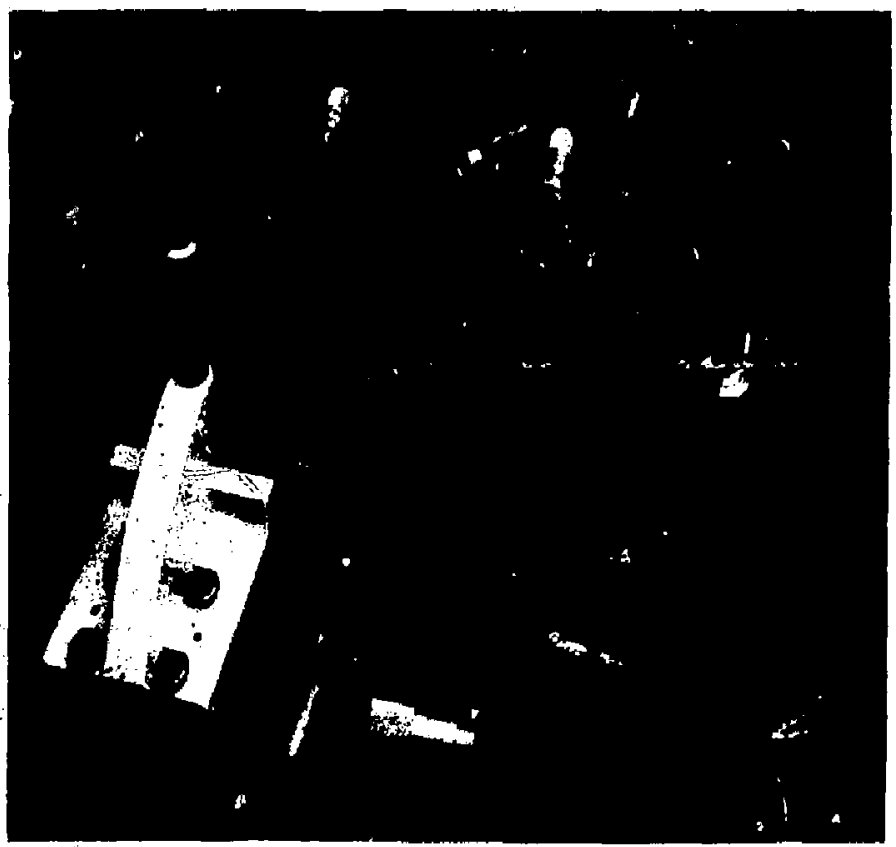

I

I

I

I

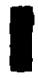

I

I

I 


\subsection{SYSTEM ALIGNMENT AND OPERATION}

\section{1 SYST EM ALIGNMENT}

The Pulse Synchronization System is based upon the heterodyne detection of signals, and must therefore be carefully aligned and maintained. A detailed alignment procedure fol.ows and must be explicitly followed each time. Although tweaking of a singie component may appear to restore operation, the sensitivity and/or resolution may be severely affected.

\section{Mode Locked Laser}

The laser cavity is formed by a 1 meter radius of curvature rear mirror, and a front flat partially transmitting mirror. The front mirror is wedged to avoid etalon effects between surfaces. This wedge causes beam steering and a second $s$ tellite beam. The rod exhibits thermal focusing and the cavity has been set up for operation from 18 to 24 amps lamp current. A nominal setting of 20 amps should be maintained. The AO cell is located between the rod and front mirror. A Brewster's stack polarizer, intracavity shutter, and $1 \mathrm{~mm}$ etalon are located between the rod and rear mirror. A mode limiting aperture is attached to the front mirror mount. IT IS IMPORTANT TO REMEMBER THAT ALL THESE COMPONENTS TILT OR TRANSLATE THE OUTPUT BEAM CHANGING SYSTEM ALIGNMENT. THEREFORE, THE LASER MUST BE ALIGNED, OPTIMIZED FOR MODELOCKED OPERATION AND THEN LEFT ALONE.

The laser should be aligned for maximum power output with the mode. aperture open and $A O$ cell set approximately for the Bragg angie. The mode aperture should be closed down and center ed repeatedly for maximum power 
using the p:ovided tool. 'This will center the apirture. Thi ctalon should be rer oved during this first alignment. Follow the Qua:atronixs manual for adjustments of the polarizer, AO cell, and Bragg angle. The etalon should be inserted and adjusted for maximum output power. This is accomplished by using the required ball drive through the rear mirror and hole in the intracavity shutter enclosure,

The output pulse should now be monitored through the rear mirror using a 50 picosec risetime detector and sampling plug-ins. The modelocker should be set to $1: 5$ watts forward power by the adjustrnent screw on the rear panel of the driver box. Cable lengths may need to be varied to reduce the reflected power. The drive frequency and Bragg angle of the AO cell should be simultaneously adjusted for minimum pulsewidth. This should be approximately 250 picosec FWHM. Centering of the beam in the mode aperture, output power, etc. should be rechecked as movement of the $A O$ cell causes beam translation. The pulsewidth appearance should be a smooth Gaussian curve, (Reducing the mode aperture causes a power reduction but provides this smoothing.) Changing the drive frequency should cause a slow broadening of pulse on either side. Secondary peaks or other anomolies indicate improper adjustment and alignment.

The modelocked output should now be stable with 250 picosec FWHM, with $500 \mathrm{~m}$ watt average power output with a lamp setting of 20 amps. The laser cover should be replaced and the laser left alone, except for occasional drive frequency adjustments.

\section{Outrit Beam Collimation}

The modelock'su laser output passes through a four times (4X) beam expander. It exits at approximately a $4 \mathrm{~mm}$ diameter. Thi s beam expande: must be centered on the beam and the mount locked down. The objective lens moves back and forth within the barrel to achieve collimation, A locking ring must first be loosened. A very thin shearing plate is used to set collisisation. 


\section{External Polarizer and Half-Wave Plate}

The laser output is polarized by an internal Brewster's stack polarizer. However, the rod thermal birefringence destroys this açoss the beam diameter. An external polarizer is required to restore the polarization purity required. With the system polarizer, halfwave plate, and polarizing beamsplitier removed, a calibrated polarizer in a rotational mount is inserted into the beam and adjusted for best extinction. The extinction will not be complete, and will be best only at the cenier of the beam. The system polarizer is inserted in its mount and rotated until total extinction across the beam results. The laser output is now a clean vertical output polarization. The calibrated polarizer is rotated exactly 90 degrees. The half waveplate is inserted and adjusted to best extinction. The beam has been rotated by 90 degrees and is now horizontal. It will now pass through the output polarizing beamsplitter and become the signal beam. By turning this waveplate small amounts, a small vertical component is developed and it passes into the $L O$ arm becoming the $1, O$ beam.

\section{Polarizing Beamsplitter}

The beam exiting the polarizer-waveplate fixture passes through a polarizing beamsplitter and its $s$ and $p$ components are split irto the respective signal and LO arms. An auxiliary centering stop with outer dimension matching the beamsplitter face, and a hole or aperture matching the size of the beam, allows the beamsplitter vertical, and horizontal positions to be adjusted. The beamsplitter rotation about a vertical axis is adjusted to give maximum extinction in the LO path. Beam height is 8.0 inches. A tilt adjustment is also provided and is used to adjust the beam parallel to the table at 8.0 inches height. The auxiliary stop is repositioned to the LO side face to facilitate adjustment of the retro return.

\section{LO Folding Mirror - Retro Tracking Adjustment}

During system operation, the dithering retro as sembly must track over $50 \mathrm{~cm}$ along the rail. The folding mirror and retro must be adjusted to provide this tracking. The dual beam assembly is removed from the rail and 
chopper positioned for beam throughput. Increase the LO arm power by rotating the half waveplate, to facilitate alignment. Return to initial position when finished. The folding mirror is initially adjusted to provide a beam parallel to LO rail at a height of 8.0 inches ( 7.5 inches above rail top). An iris is closed on the front of the retro and beam observed with an IR viewer. Both the retro and folding mirror are adjusted to maintain this centered posi. tion as the retro assembly is translated back and forth along the rail. The iris is opened and the retro return adjusted to pass back through the cube beamsplitter target. . Insure tracking. Observe the retro return on the detector side of the cube beamsplitter.

Insert the dual beam assembly back on the rail and adjust the flats for 45 degrees. it may be necessary to I'RANSLATE the folding mirror and retro by equal amounts to offset the translation of the flats. Observe the two existing beams at the second surface of the first flat (chopper side). Adjust the folding mirror so that the two beams are equi distant from the optical coating line on this surface. Slight adjustments should be necessary. Adjust retro for tracking and return through cube target.

\section{Detector Arm Folding Mirror}

The LO beam is now in the detestion arm. Adjust the folding mirror - for a beam parallel to the detector rail at a height of 8.0 inches. Close down the iris in front of the detectors. Use a small aperture and align the detector assembly to the beam. Visually assure that the detector cylindrical body appears level and parallel to the beam. A large misalignment can result in reflections from the walls of the detector assembly that inay appear to be in alignment.

Remove the two detectors from the mounts. Insert the two nlexiglass targets with metal crosshairs. Adjust the detector assembly to center the observed beams on the crosshairs. The detector assembly is comprised of a focusing lens, Wollaston prism and two detectors. The Wollaston prism splits and p polarizations and puts each on a detector. The angle between the beams is 5 degrees. Vertical, horizontal, and rotational adjustments are provided. In addition, the beam separation is adjusted by translating the 
detectors towards or away from the Wollaston prism. Turning the rear knurled collar accomplishes this.

This completes the preliminary alignment. REMEMBER TO REMOVE THE DETECTOR TARGETS AND REPLACE DETECTORS.

\section{Dual LO Alignment}

This is the final alignment step. Unless those proceeding this have been carefully accomplished there is no reason to proceed. For successful heterodyne detection we must match phase fronts, beam size, and have colinear beams at the detector surfaces, As a further complication, this system has two separate LO beams, which must be simultaneously aligned with the same signal beam. The following procedure must be followed to accomplish this.

The preamp monitor output on the electronics panel should be connected to an oscilloscope. The chopper reference should be used as an external trigger. Turn on the chopper (switch on the black box in close proximity). If the preceding alignment was followed, a chopped signal waveform should be seen on the oscilloscope. Using a peace of folded paper, block one of the LO beams. A chopped waveform should still be observed. There are three adjustments to the detector assembly that will be required. These are tilt about the horizontal, rotation about the vertical, and rotation of the whole detector body about the bearn axis. Rotate the body about the beam to effect a quasi straight line display. Tilt about the horizontal, first one way and then the other. The chopper waveform will return, firsi above, then below zero. We wish to achieve symmetry of the se excursions above and below. Small rotations of the body will eventually achieve the desired symmetry. Repeat for rotation about vertical. Continue until both tilt about horizontal and rotation about vertical, both + and -, achieve the desired symmetrical excursions, with a straight line display at middle settings. This has aligned one LO beam to the detectors and balanced their amplitudes.

Unblock the second LO beam. The only adjustments are made to the beam splitting llats. Only very minor adjustments are required. By adjusting the flats about vertical and 45 degrees we are aligning the second LO beam to 
the first. It is essential that they do coincide. Carefully, with small adjustments restore a straight line display. (Unblocking second LO beam should result in a chopped waveform on the scope).

We have now aligned the two LO beams to the detectors and each other. The last alignment item is adjustment of the chopper. The chopper alternately blocks one or the other of the LO beams. However, there are transitional regions where both beams exist. (One coming on, one going off). It is necessary to adjust the duty cycle of the chopper to less than 50 percent. The dual blades allow variable spacings. The spacing is empirically adjusted to remove the transistional periods. Symetrical waveform is set by the height adjustment on the chopper. Horizontal adjustment has little or no effect. This completes the system alignment. The remaining adjustments are operational. Figure $3-1$ shows the sketches of the various naveforms desired.

\section{2 SYSTEM OPERATION}

Once, stable modelocked laser operation has been arhieved, and a careful alignment performed, the system is ready for operation. A brief review of the theory, and usage is required to provide direction for the following operational adjustments.

The modelocked pulse train is amplitude divided and the signal portion transverses the amplifier train, is retro-reflected, and returns. It suffers attenuation and a time delay. It is heterodyne detected with a LO pulse train $\therefore$ that has been shifted in frequency by the motion of the dithering retro mirror. Only when the pulse trains overlap is there a detected signal. This positional overlapping creates a heterodyne response curve for each LO beam. Since there are two LO pulse trains, themselves having a relative delay, a response curve like that shown in Figure 3-2 results, IT IS IMPORTANT TO REALIZE THAT A CHANGE IN THE MODE SPACING OF THE MODES SUMMING TO THEŚE PULSE TRAINS SHIFTS THESE CURVES. IT IS THEREFÓRE, IMPERATIVE THAT THE MODELOCK DRIVER NOT BE ADJUSTED DURING A MEASUREMENT. The chopper alternately selects one LO beam then the other. This results in a square wave amplitude modulation of the detected heterodyne signal. Refer again to Figure 3-2. It can be seen that for a fixed 
TILT ABOUT HOAIZONTAL
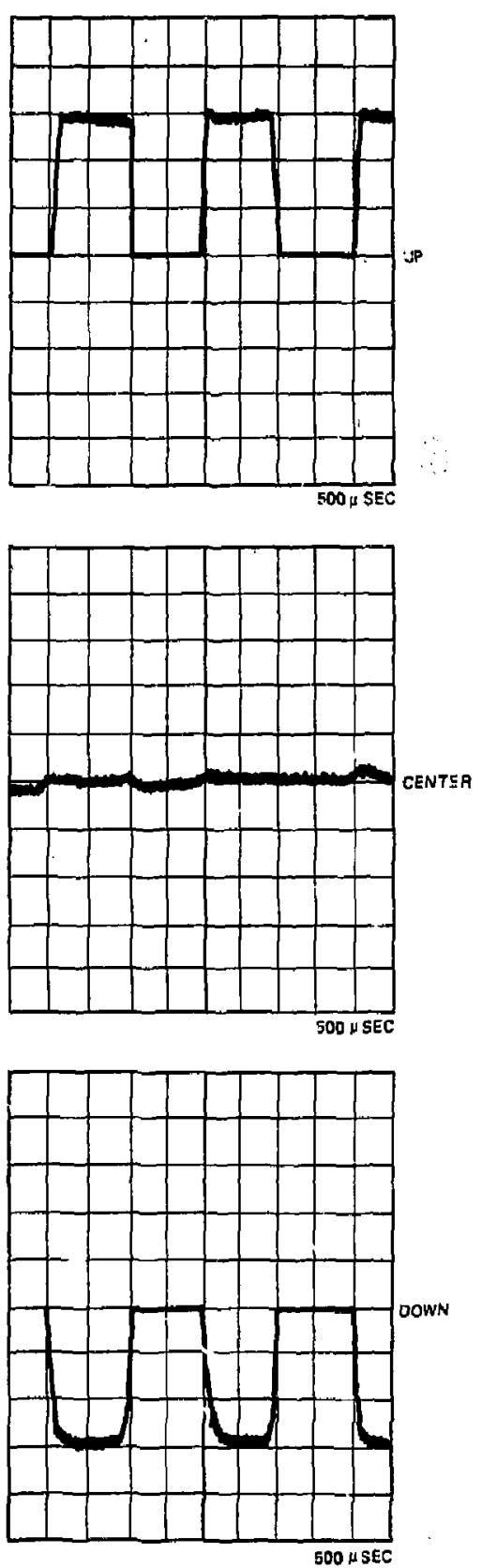

FOTATION ABOUT VERTICAL
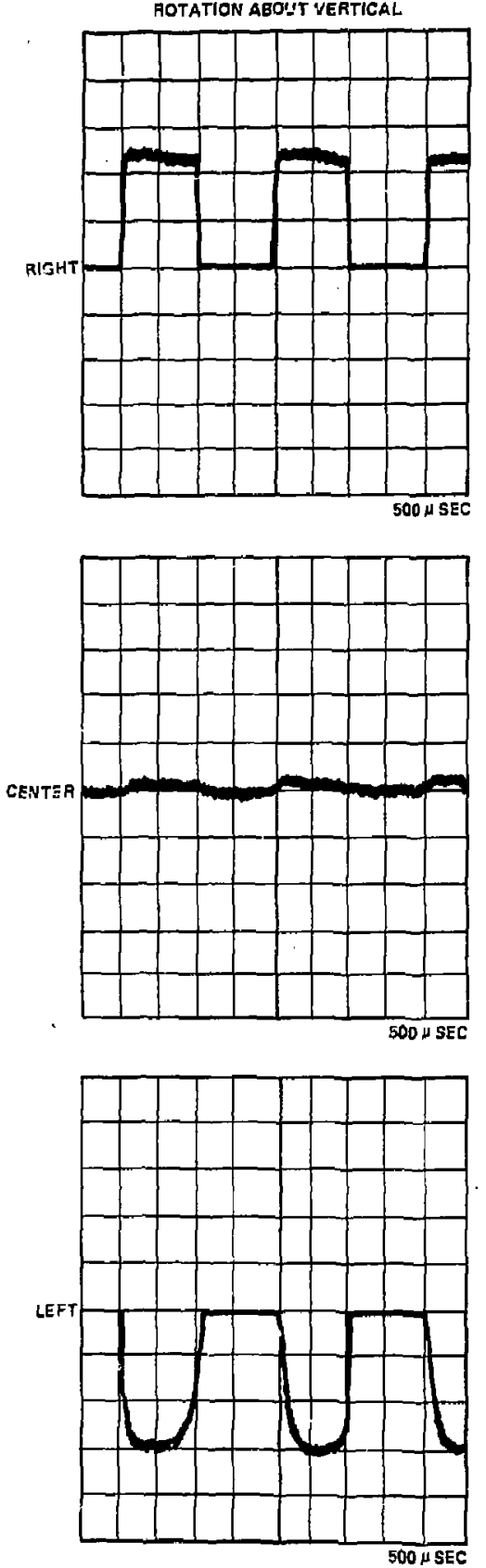

REQUIRE EOUAL MAXIRSUN EXCUASIONS

FOA MOTIONS ABOUT AXES - EOUALIZE

EXCJASIONS BY ROTATION OF THE DE TECTOA

BODY ABO'JT THE OPTICAL AXIS

Figure 3-1. Dual LO alignment detector preamplifier outputs for detector motions. 
HETAODYNE RESPONBE CUAVES

PATH LENGTH DIFFERENCE <NL (N - INT:EER)

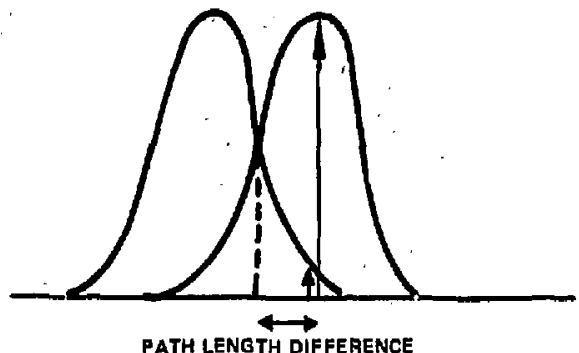

PATH LENGTH DIFFERENCE

PATH LENGTH DIfFERENCE * NL

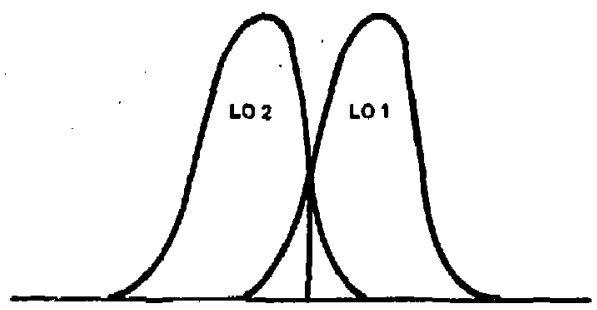

PATH LENGTH DIFFERENCE >NL

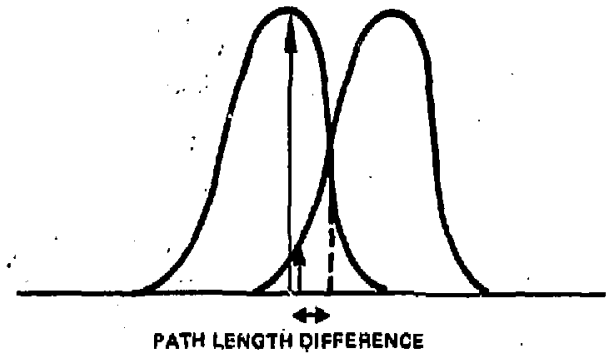

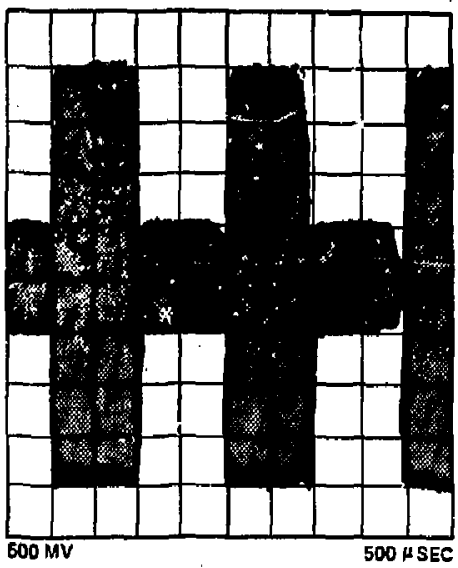

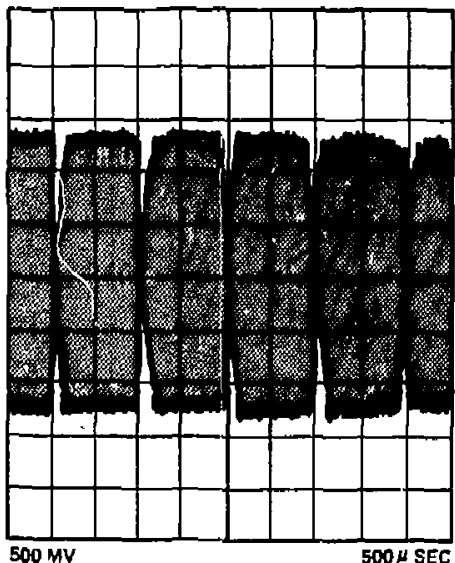

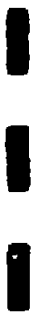
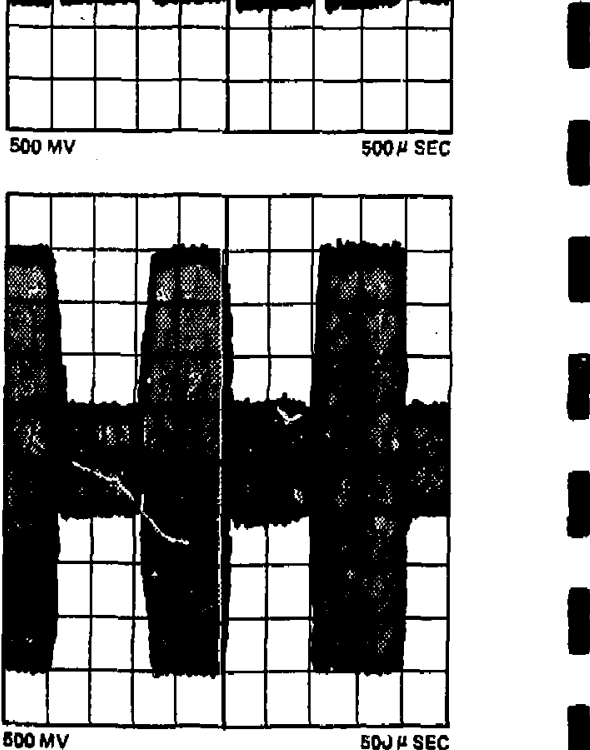

Figure 3-2. IF signal waveforms. 
relative LO spacing, when the relative path length $x$ (see Fig. 1-1) is an integral number of cavity lengths, the square wave modulation has a null. Moving off centel causes one LO response to increase and the other to decrease, and vice versa for a movement off center in the other direction. Therefore not only is the magnitude of the equalization offset indicated, but also its direction with respect to the ref arm is indicated.

\section{Initial Test for Operation}

Bef:re using the PSS on the laser amplifier chains, a bench test should be perfo ...d lu insure uplimum periormanc. $.90 \mathrm{~dB}$ attenuation. Measure the distance from the center of the polarizing beamsplitter along the folded optical path to the rear of the dither assembly. Set up a flat gold mirror at one or two times this distance. Place a filter holder and ND filters about a foot from the signal exit aperture of the pulse synchronization system. Tilt these filters so that any Fresnel reflections strike the front panel and do not re-enter the system. Starting with ND of 2 (-40 dB round trip), adjust the flat return mirror for a heterodyme return. This can be observed on on the preamp output or boost amp output and will be a very large signal. Periodically block the signal path to insure a signal is present. With the signal blocked, increase LO power by rotating the half waveplate, until the waveform on the boost amp output exhibits noise spikes on the waveform during transitional periods of the chopper. See Figure 3-1. Gradually increase the ND to a total of 4.5. At this attenuation a signal to noise of 5 or less with a p-p value of 1 volt should be present. Each time a new ND filter is added maximize the signal by tweaking the flat return mirror. Optimize the signal by rotating the output quarter waveplate.

At this point, the resolution is ready to be tested. When the alignment, LO power, and signal return have been optimized, block the signal beam, and adjust the front panel boost amp control while ronitoring the envelope detector output. Adjust it high so that the noise spikes can be seen and then gradually reduce the gain until the noise is thresholded.

Unblock the signal, and the square wave output of the envelope det should he seen. Ensure that the post amp is tuned to ref chopper frequency. 
See discussion of electronics. The PAR lockin should be turned on, and allowed to lock on to the chopped frequency. Adjust the amplitude and time constant to get a response. Displace the dither assembly so that a visible square wave is seen modulating the boost amp output. Using the lockin phase settings obtain a null on the meter. Now turn the phase switch by 90 degrees and move the dither retro assembly to obtain a null. The PAR now has been calibrated. The reference phase adjustment need not be touched again. Lock down the front part of the dither assembly and adjust the micrometer two two full turns ( $1 \mathrm{~mm}$ ). A meter deflection of 0.1 to 0.8 units should occur (for an attenuation of $10 \%$ ). Experience in alignment and operation of the system will be required to obtain the near full scale deflection.

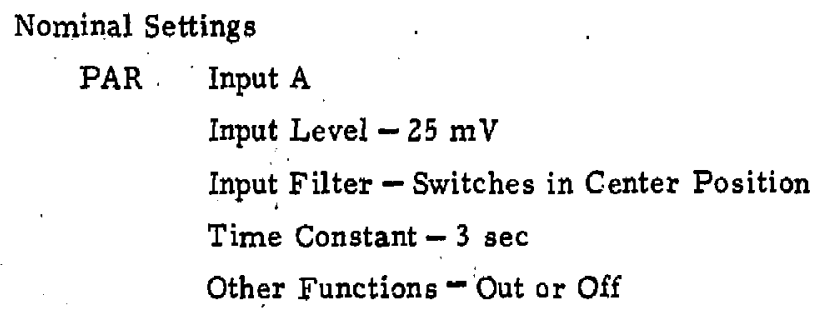

Electronic s Panel

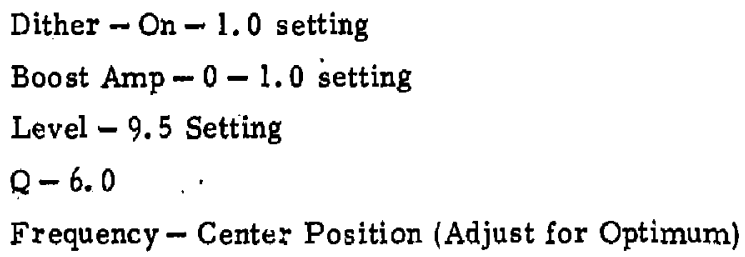

\section{Operational Usage}

Testing on the Cyclops chain showed that basically there is no difference in operation of the system. The laser power was visible on the alignment camera at the target. The output quarter waveplate was rotated for maximum observed intensity as indicated on the TV camera monitor.

Ghost signals, or retro reflected returns from certain elements up to the first.rod amplifiers must be carefully watched. In the Cyclops system many flat surfaces such a corner cubes front face, plano convex lens, proved to be a problem. These were quickly eliminated by slight adjustments to 
these componenis. Additionally beam splitters and partially transmitting mirrors caused some problems with the transmitted heams srriking mounts etc, and back scattering. Replac ement with gold front surfaces mirrors eliminated these false returns.

Operation was the same as the bench test. Once a signal is detected and optimized, set the boost amp level to threshold the background noise. The system is now operational and the first amplifier chain has been set up as the reference. By going to successive arms the meter will deflect + or indicating which way ise path must be changed. The path length equalizers are positioned to obtain a meter null on the PAR. This path is now within a faction of a millimeter to the reference arm. Although not necessary, it would be good measurement practice to periodically check the reference channel for a null. Proceed with each chain, one at a time, obtaining nulls on the meter. NO CHANGES TO ANY FUNCTION OF THE PULSE SYNCHRONIZATION SYSTEM SHOULD BE MADE DURING MEASUREMENT. ONLY THE ACTUAL PHYSICAL PATH LENGTH OF THE CHAIN BEING MEASURED SHOULD BE EFFECTED. Failure to do so changes the calibration of the reference and it must again be nulled as described above. 


\subsection{PHYSICAL DESCRIPTION}

\subsection{DESCRIPTION - CIRCUIT BOARD Al (FIGURE 4-1)}

Circuit board Al contains switching transirtors to control current through the windings of the blast and intra cavity shutter relays. Two identical circuits are provided, one for each shutter. Base drive is provided by a manual switch on the front panel. When this switch is closed, base current flows, turning on the transistor and illuminating the LED indicator. This causes current to flow in the relay windings pulling down their switch contacts. This in turn energizes the shutter, opening it. Additionally, a status light goes frum green to red. External control is provided to shut the shutters, for instance during a chain firing pulse. An external short or low impedance removes the base drive current turning off the LED indicator and transistor de-energizing the relay and shutter, which then closes. 


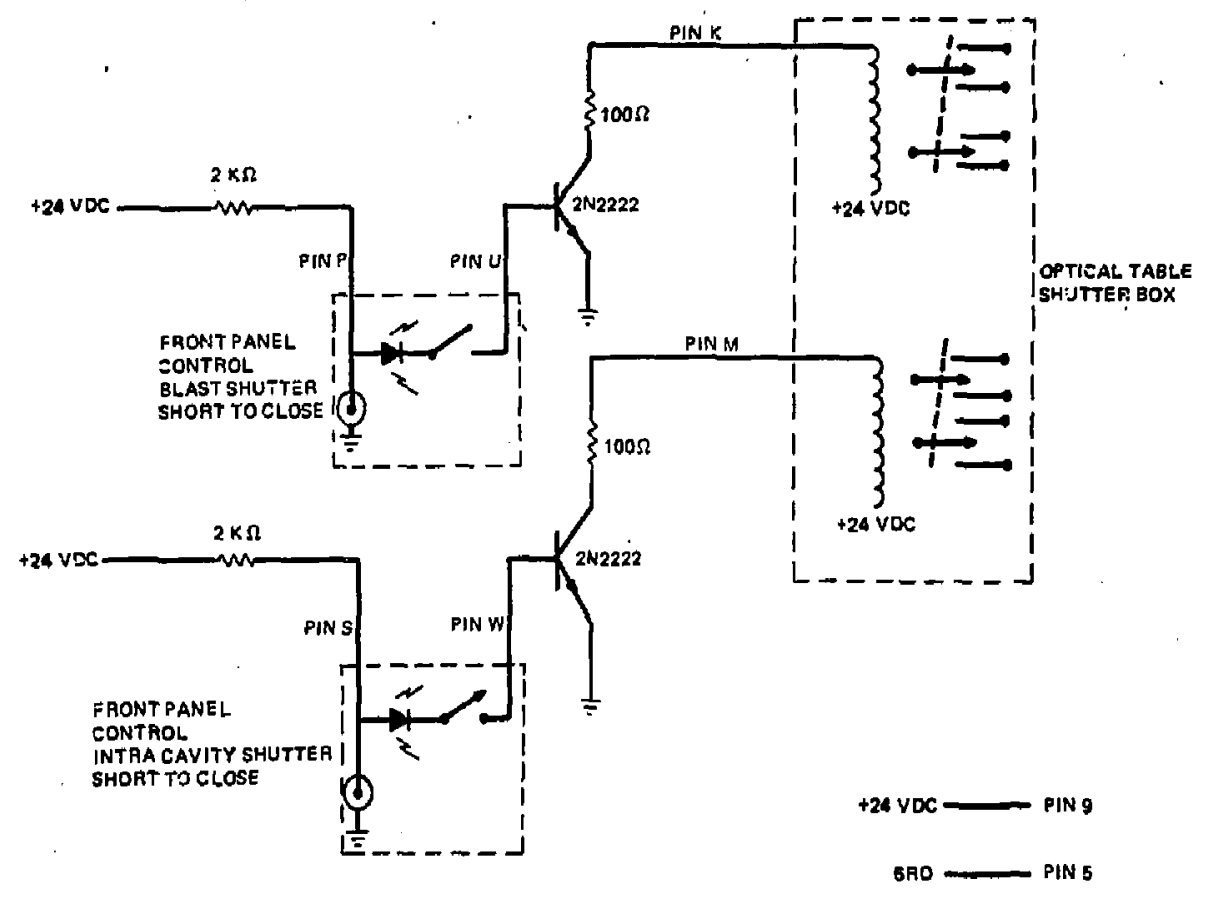

a.

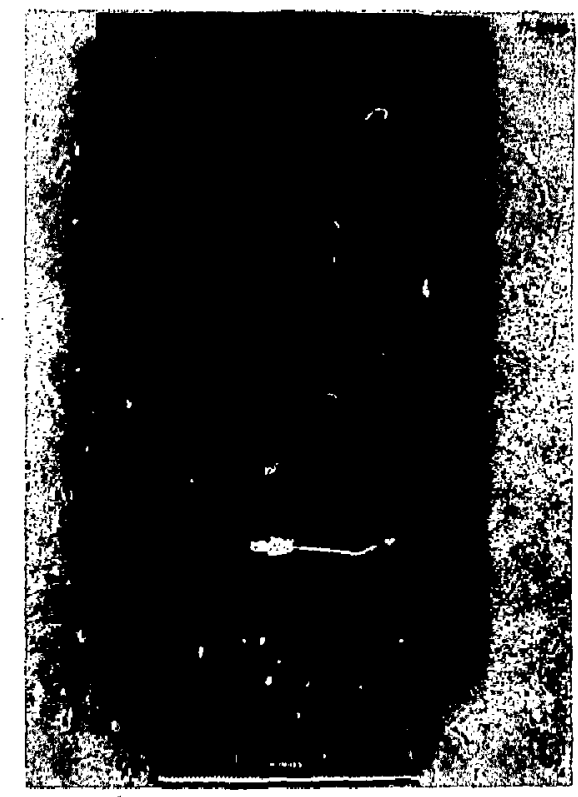

b.

Figure 4-1. Electronics - Circuit Board A1-Shutter Control. 


\subsection{DESCRIPTION - CIRCUIT BOARD A2 (FIGURE 4-2)}

Circuit board $A 2$ is the post filter. It is the final filter centered at the chopped 10 frequency, to increase the dynamic reserve of the lockin amplifier. It is frequency tunable, and its $Q$ (bandwidth) can be varied. Frequency tuning is provided by the two ganged potentiometers, and $Q$ is varied by the potentiometer in the overall feedback loop. Normal operational settings are center scale on frequency and a 6.0 setting on $Q$. By feeding the reference signal into the envelope detector monitor using a 100.1 attenuation, the frequency can be optimized by a maximum meter deflection on the lockin amplifier meter. 


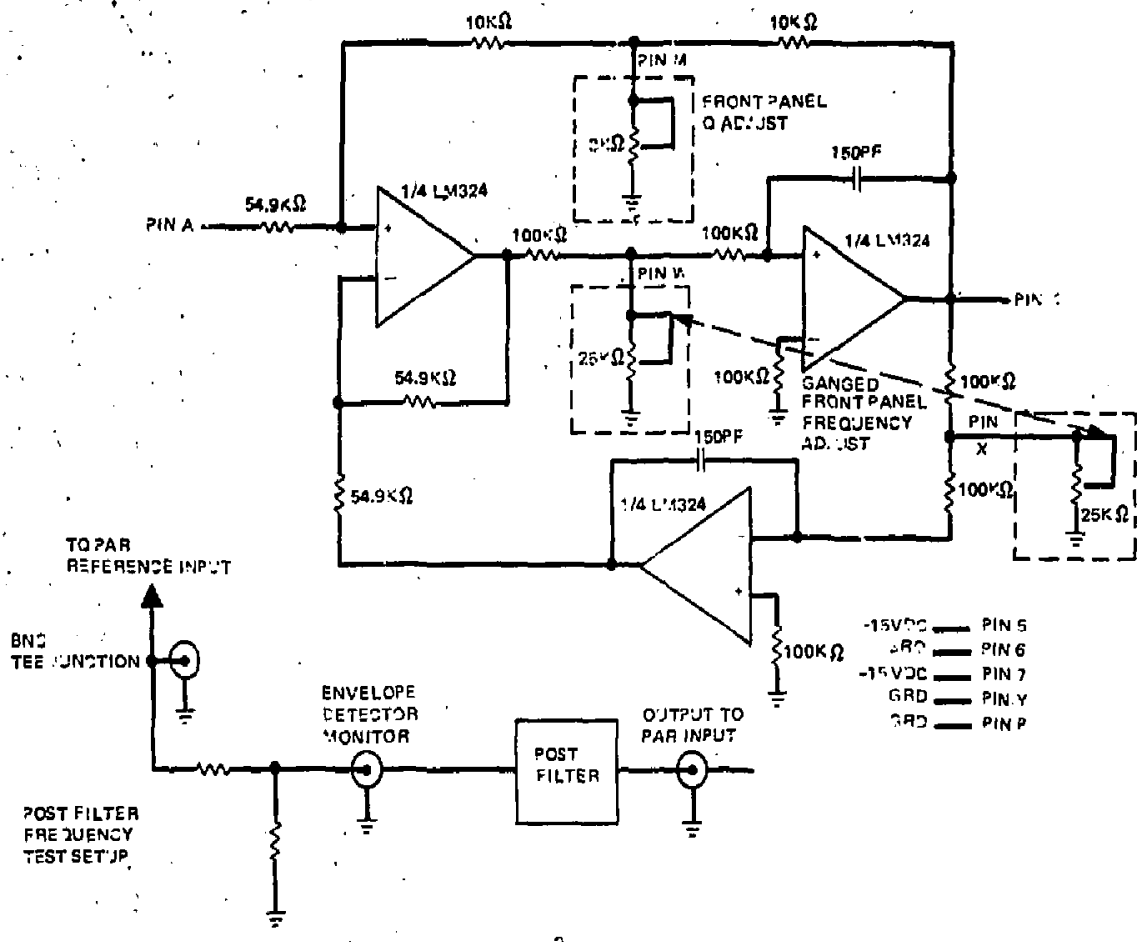

a.

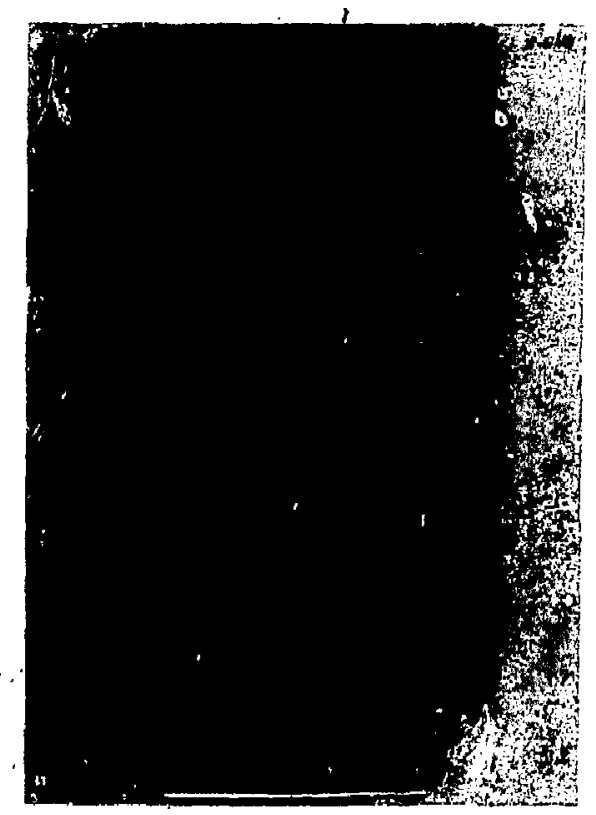

b.

Figure 4-2. Electronics - Circuit Board A2 - Post tuned filter. 


\section{3 DESCRIPTION - CIRCUIT BOARD A3 (FIGURE 4a)}

Circuit board A3 contains three functions. These are the boost amplifier, the signal level monitor circuit, and the AM demodulator or envelope detector.

The boost amplifier contains three (3) amplifying stages. The first two amplifiers can be set at gains of one (1), ten (10), fifty (50), and one hundred (100) with a switch assembly. A closed cordition is synonomous with on. The third stage is continuously variable by a potentiometer located on the front panel.

The signal is split and isolated by buffering stages. A long time constant envelope detector followed by a unity gain buffer drive a signal level output meter. The variable adjustment is for calibration of the meter to indicate half scale when 1.0 volts $\mathrm{p}-\mathrm{p}$ signal is present.

A short time constant envelope detector follows the heterodyne detected signal, amplitude modulated envelope. This demodulated output passes through a potentiometer. This pot controls the signal level into the lockin amplifier and is adjusted to avoid an overload condition. It is located on the front panel.

The gain on the boost amp is used to maintain the noise below the forward conduction voltage of the envelope detector. In this manner the noise is thresholded. Any signal with $\mathrm{S} / \mathrm{N}$ greater than 1 wil then be received by the PAR. Normal operational settings are:

1. Boost Amp - 0.1

2. PAR Inpuit Level -9.5 

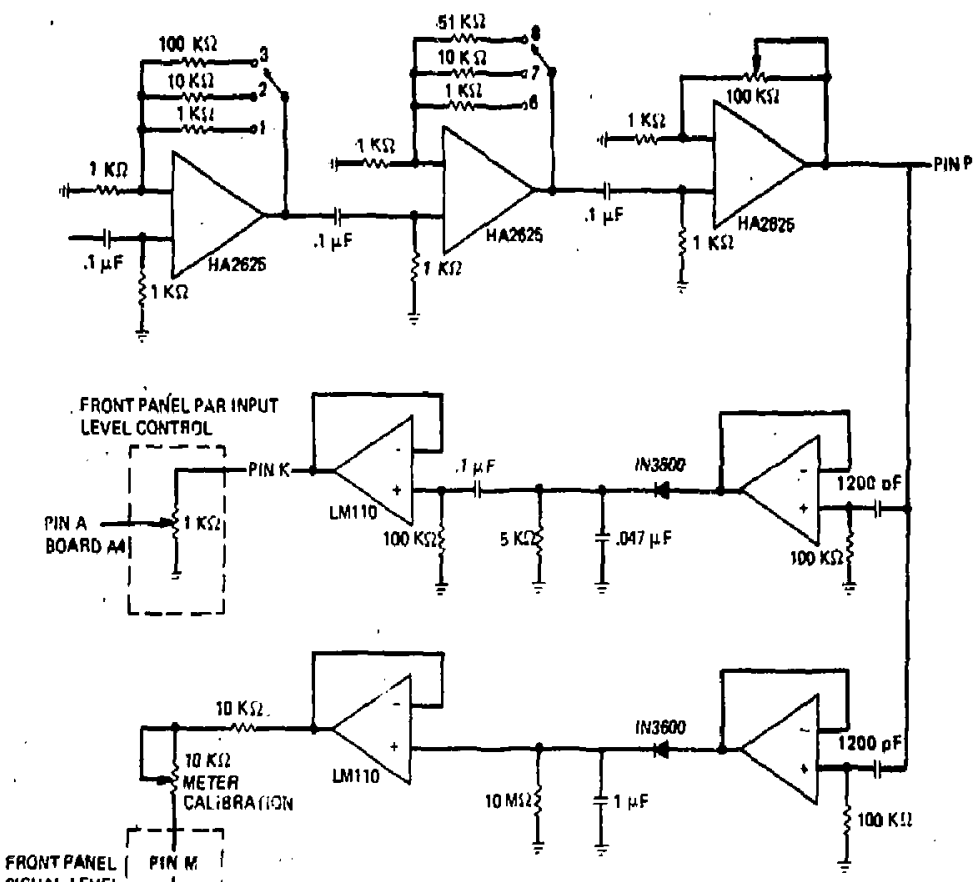

FRONT PANEL I PIN $\mathrm{K}$ । SIGIHAL LEVEL MONTTOR.

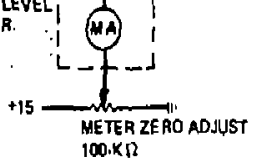

D.Kn

a.
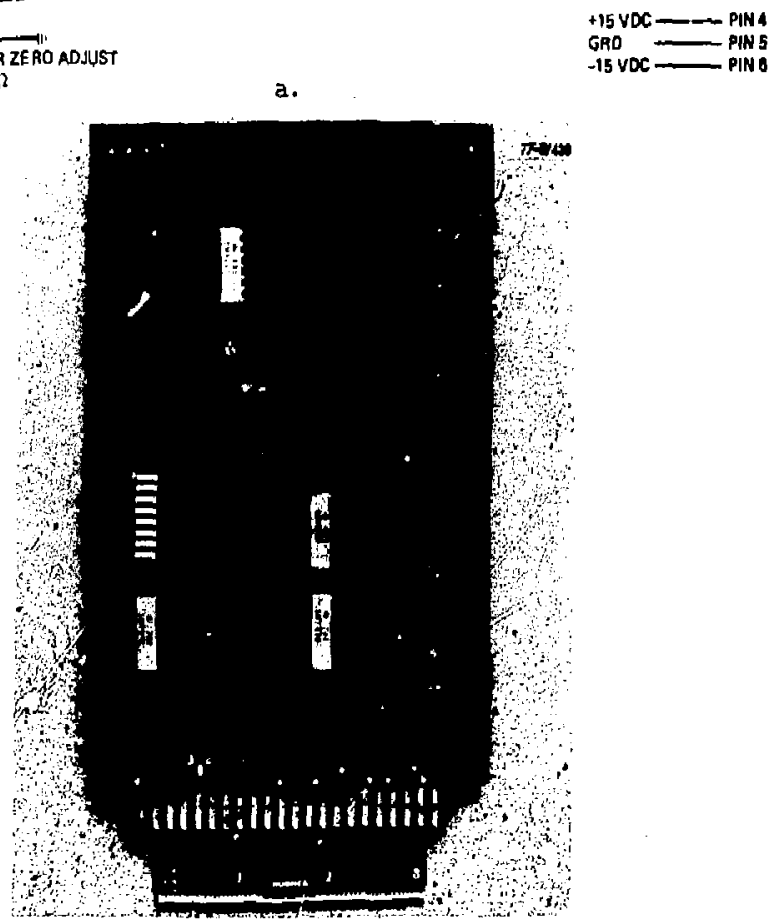

b.

Figure 4-3. Electronics - Circuit Board A3 - Boost Amplifier and Envelope detector. 


\subsection{DESCRIPTION - CIRCUIT BOARD A4 (FIGURE 4-4)}

Circuit board A4 is designed to remove low frequency chopped LO components and transitional transients of the chopping blade. It is comprised of four (4) stages of active highpass filters. Each filter is a $3 \mathrm{rd}$ arder active high pass filter with cutoff at $30 \mathrm{kHz}$. The overall four stage response is down $-12 \mathrm{~dB}$ at $30 \mathrm{kHz},-70 \mathrm{~dB}$ at $10 \mathrm{kHz}$. 

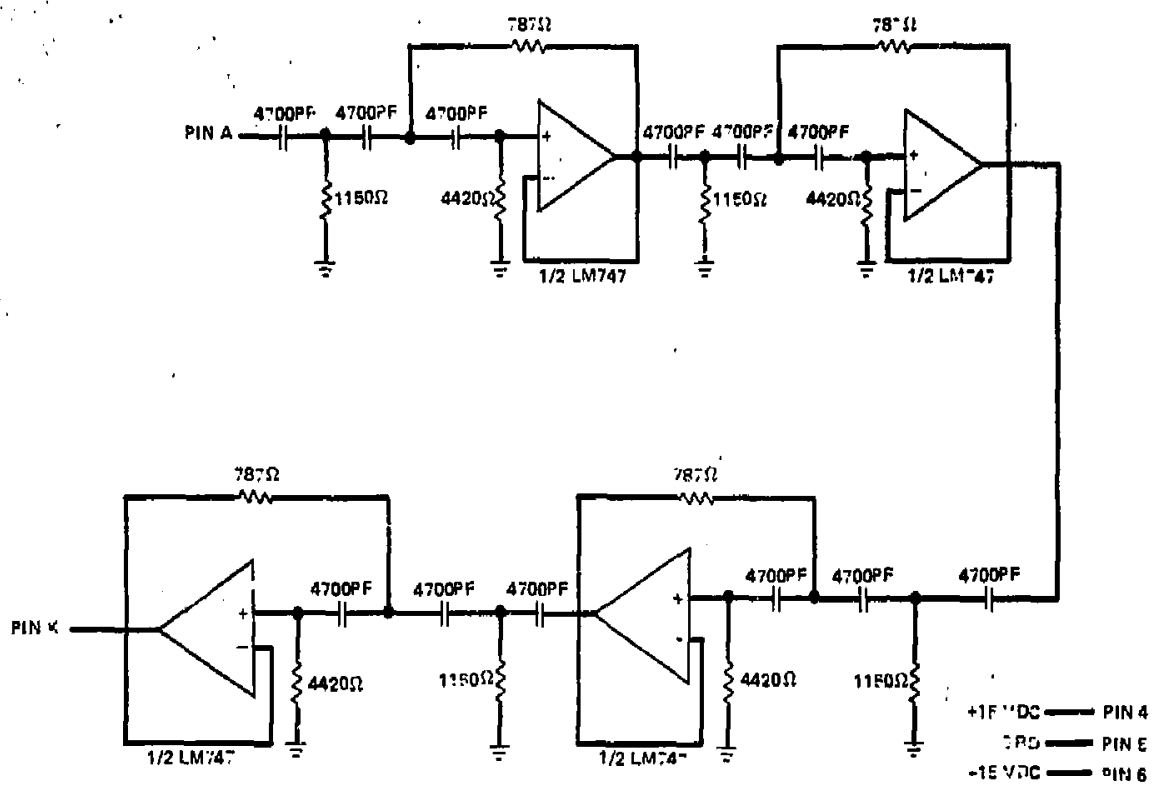

a.

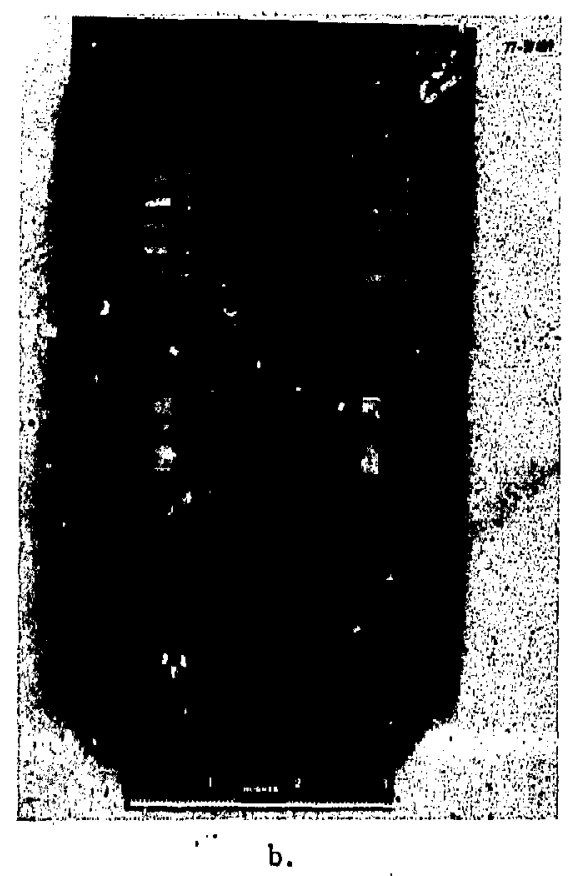

it

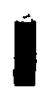

南
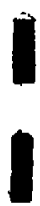

$\because$

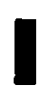




\subsection{DESCRIPTION - CIRC:ITT BOARD A5 (FIGURE 4-5)}

Circuit board $\mathrm{A} 5$ is a switched sine or triangle function generator. Symmetry and waveforn are fixed sinewave adjustments. Amplitude is controlled by the $50 \mathrm{~K} \Omega$ pot, frequency by the $100 \mathrm{~K} \Omega$ pot, and switched between sine and triangle operation by an on board switch. Actual operation will be either sine or triangle depending on which provides the best results. (Minimum turnaround time - low frequency periods.) The output is split, one serving as a constant reference signal at the front panel, the other goes to a front panel pot and on to the dither driver. 


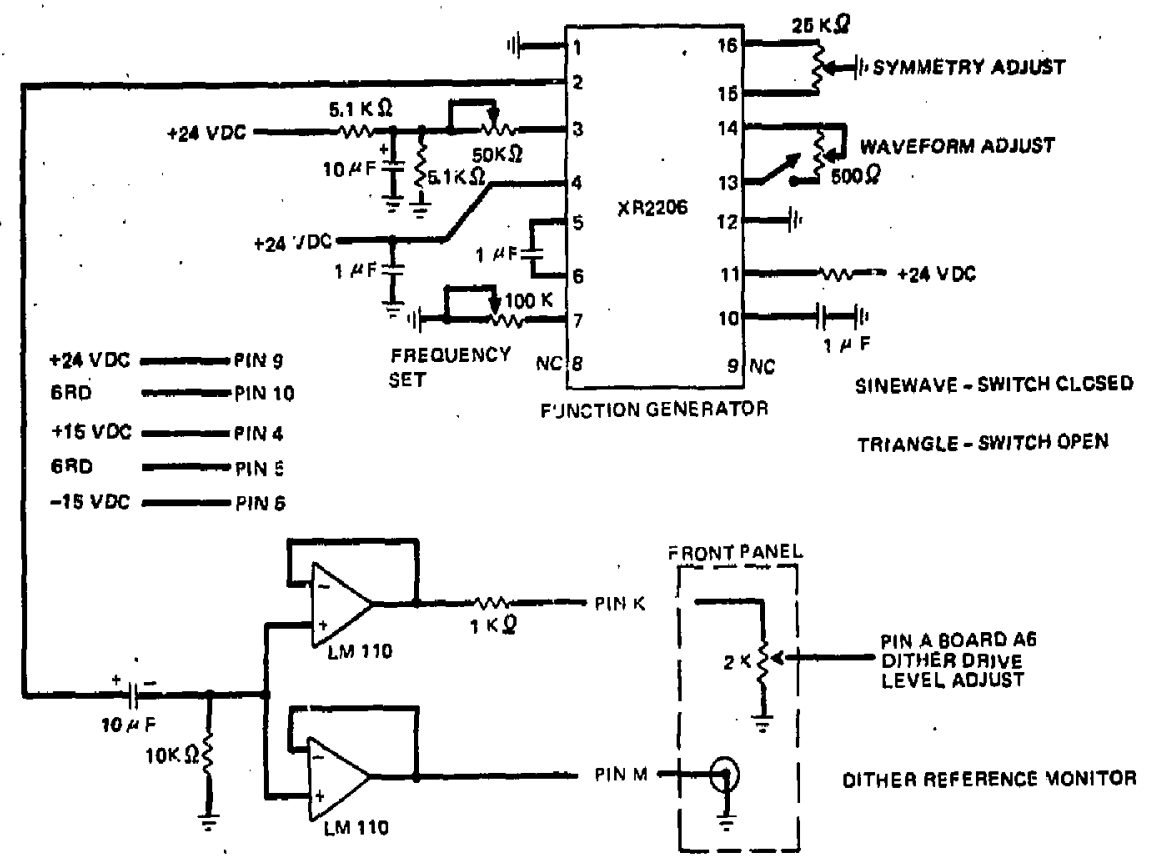

a.

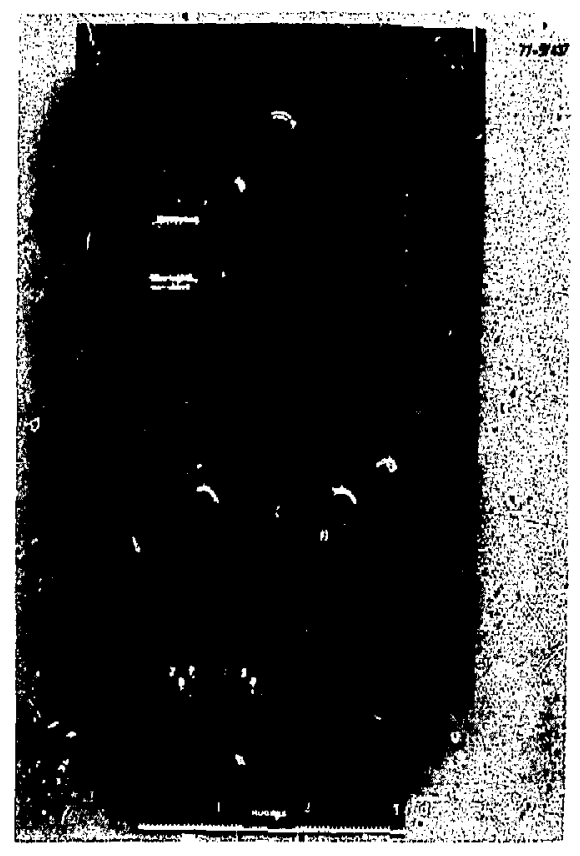

b.

Figure 4-5, Electronics - Circuit Board A5-Dither Oscillator. 


\section{6 DESCRIPTION - CIRCUIT BOARD A6 (FIGURE 4-6)}

Circuit board $\mathrm{A} 6$ is the dither driver. It receives a controlled drive signal from the front panel dither adjustment. It is a differential driver. A drive current monitoring circuit is not used. It is comprised of a commercial 6 watt dual audio range amplifier chip. 


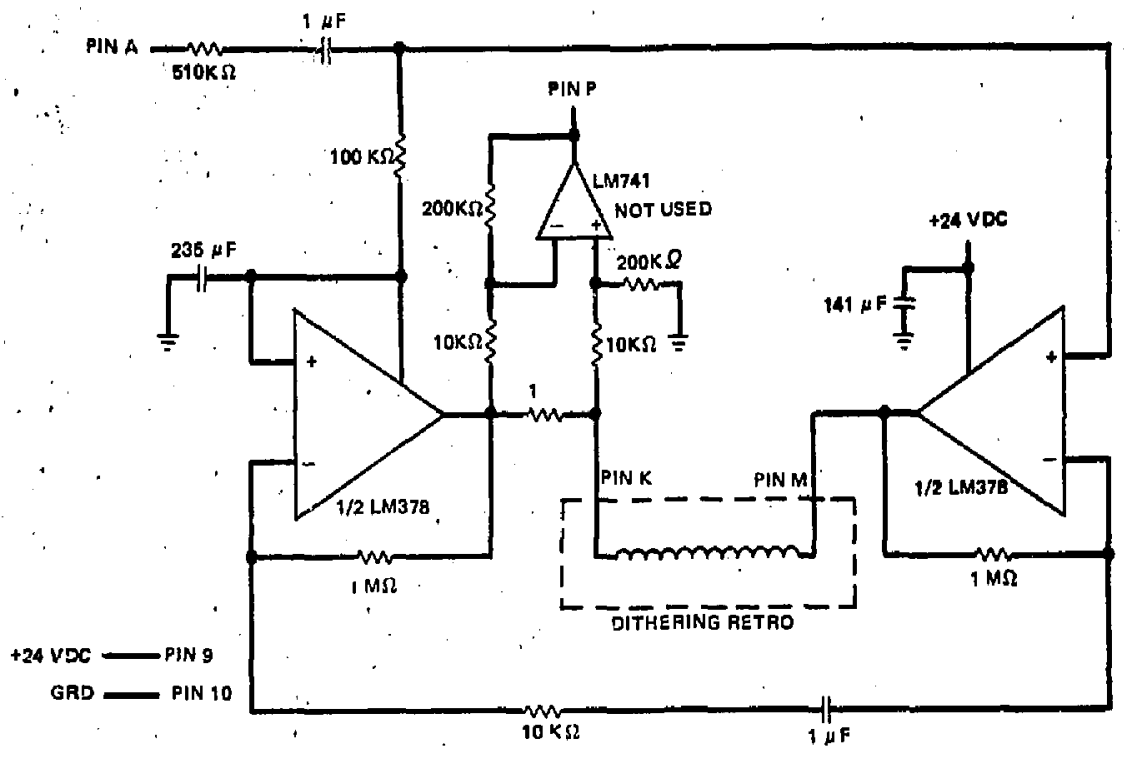

a.

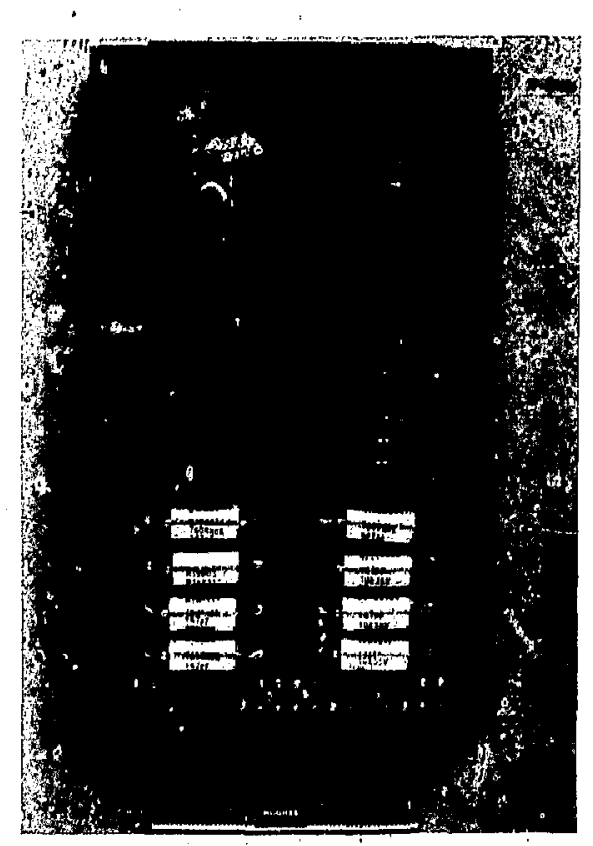

b.

Figure 4-6. Electronics - Circuit Board A6 - Dither Drive, 


\subsection{DESCRIPTION - CIRCUIT BOARD A7 (FIGURE 4-7)}

Circuit board A7 is a three voltage output supply. +15 regulated output drives the dither indicator light. $+24 \mathrm{~V}$ large current provides that required for the dither driver. The unregulated voltage is used to power the blast shutter solenoid. 

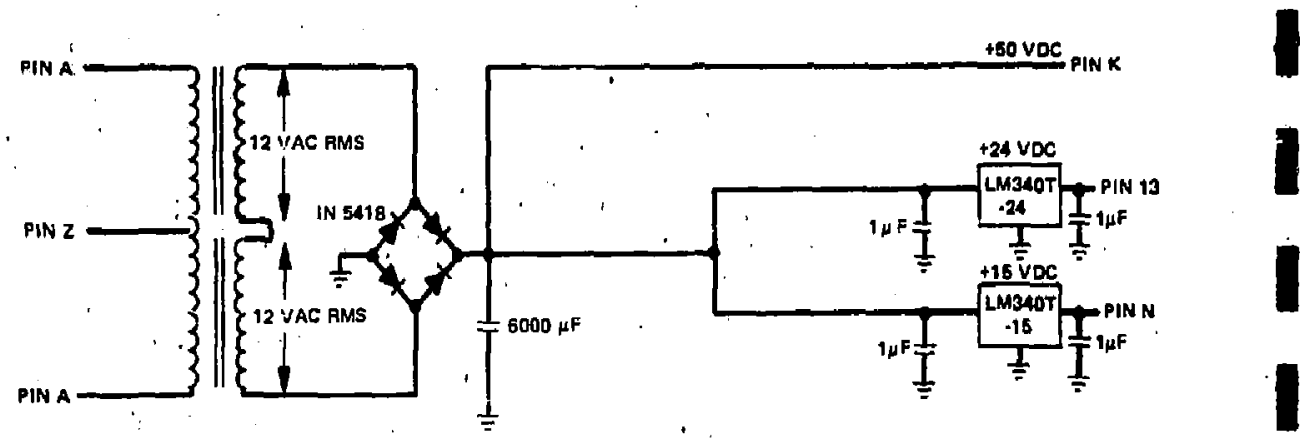

a.

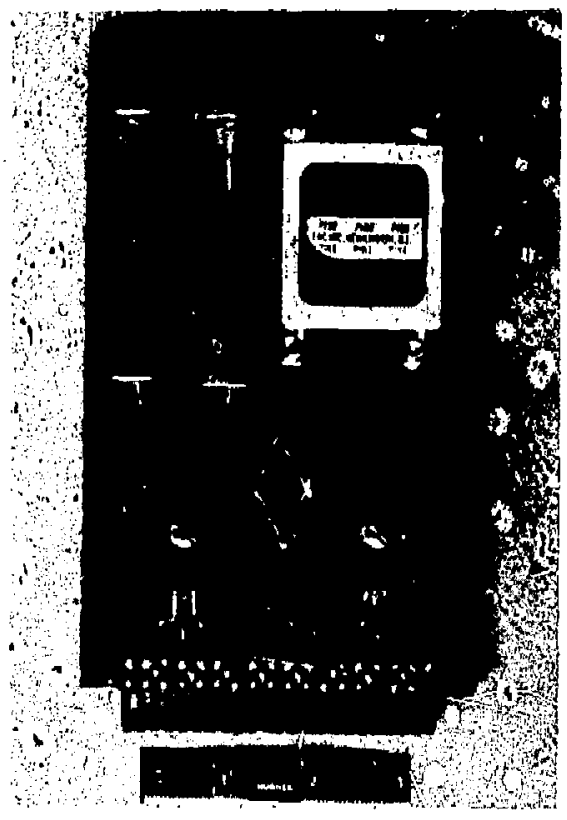

b.

Figure 4-7. Electronics - Circuit Board A7- +50 VDC, +24 VDC, +15 VDC Supply. 


\section{8 DESCRIPTION - CIRCIIT BOARD A8 (FIGURE 4-8)}

Circuit board $A B$ is a dual \pm 15 regulated supply. Two commercial 24 VDC supplies are used to provide the \pm 24 V'DC input to the LM 125 \pm 15 tracking regulators. The output from one regulator powers the electronics while the other supplies bias for the detectors and preamplifier. 


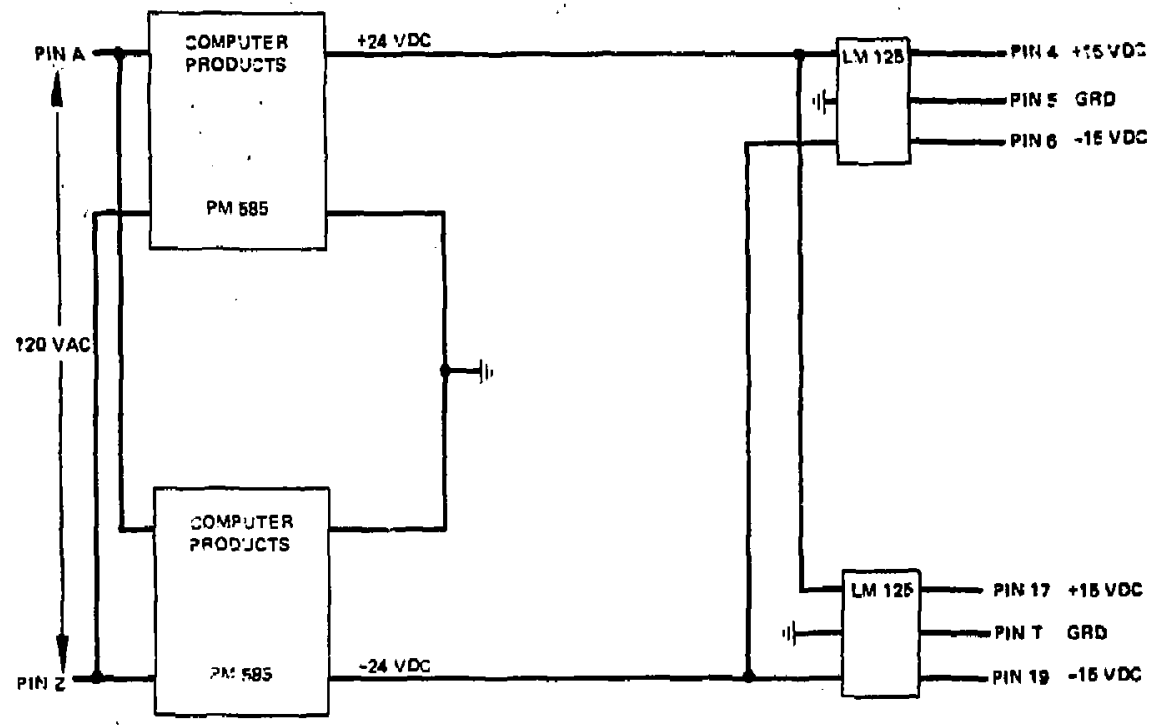

a.

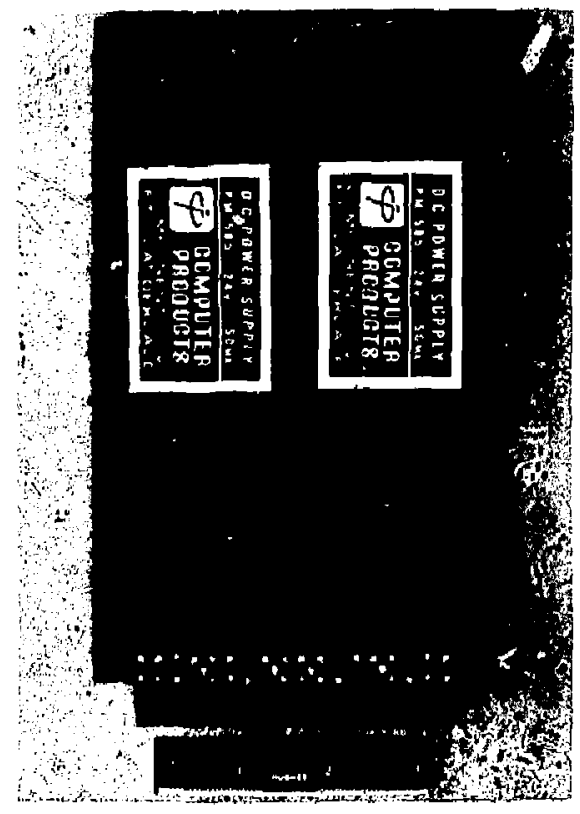

b.

Figure 4-8. Electronics - Circuit Board A8 - Dual \pm 15 VDC Supply. 


\subsection{DESCRIPTION - CIRCUIT BOARD A9 (FIGURE 4-9)}

Circuit board A9 is a dual +24 VDC regulated supply. Commercial 28 VDC supplies are regulated to 24 VDC. One output supplies the electronics. The other provides power for the blast shutter relays and circuits. 


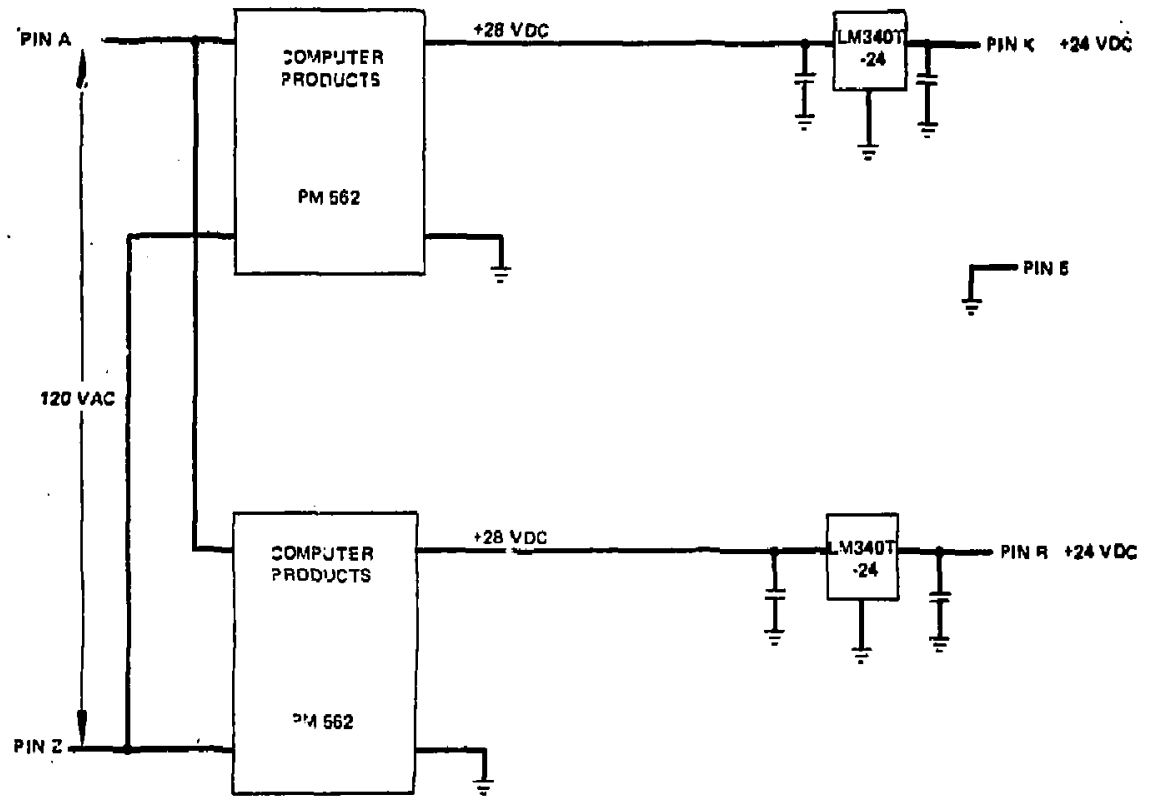

a.

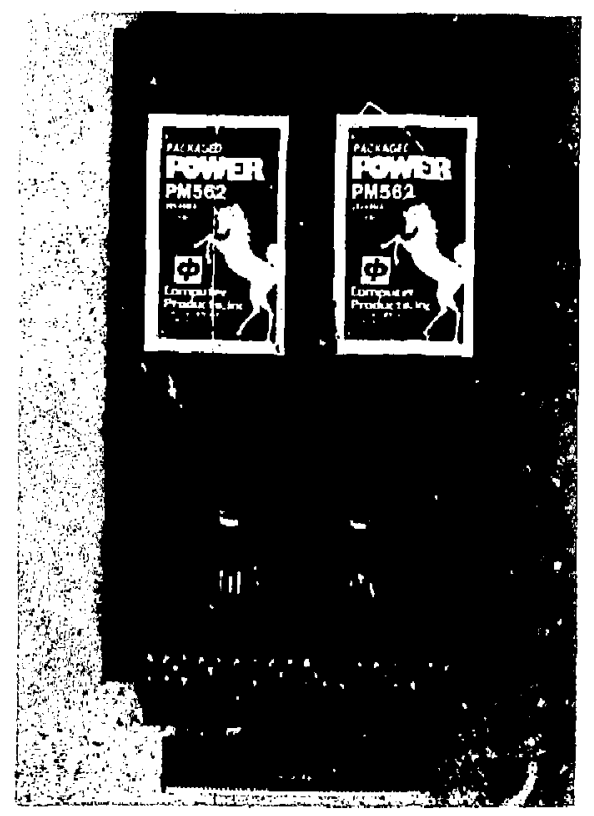

b.

Figure 4-9. Electronics - Circuit Board A9 - Dual +24 VDC Supply. 


\section{10 DESCRIPTION - BLAST SHUTTER AND INTRACAVIT $Y$ SHUTTER OPERATION (FIGJRE 4-10)}

An intracavity shutter is located within the laser resonator to make the system "eye" safe without required system shut off. Thermal stabiliza. tion is required for best modelocking, so that the lamp stould remain on except during extended inoperative periods. The intracavity shutter should be opened during systern operation to allow lasing. A red light indicates an open shutter or lasing condition, while the green indicates a closed shutter and nonlasing condition, Remote operation of this shutter is possible by shorting or switching a low impedance across the provided BNC connector. This closes the shutter.

A blast shutter covers the system output aperture to protect the system from spurious returns during firing of the SHIVA chains, that might damage components or detectors. Its operation is identical to the intracavity shutter described above.

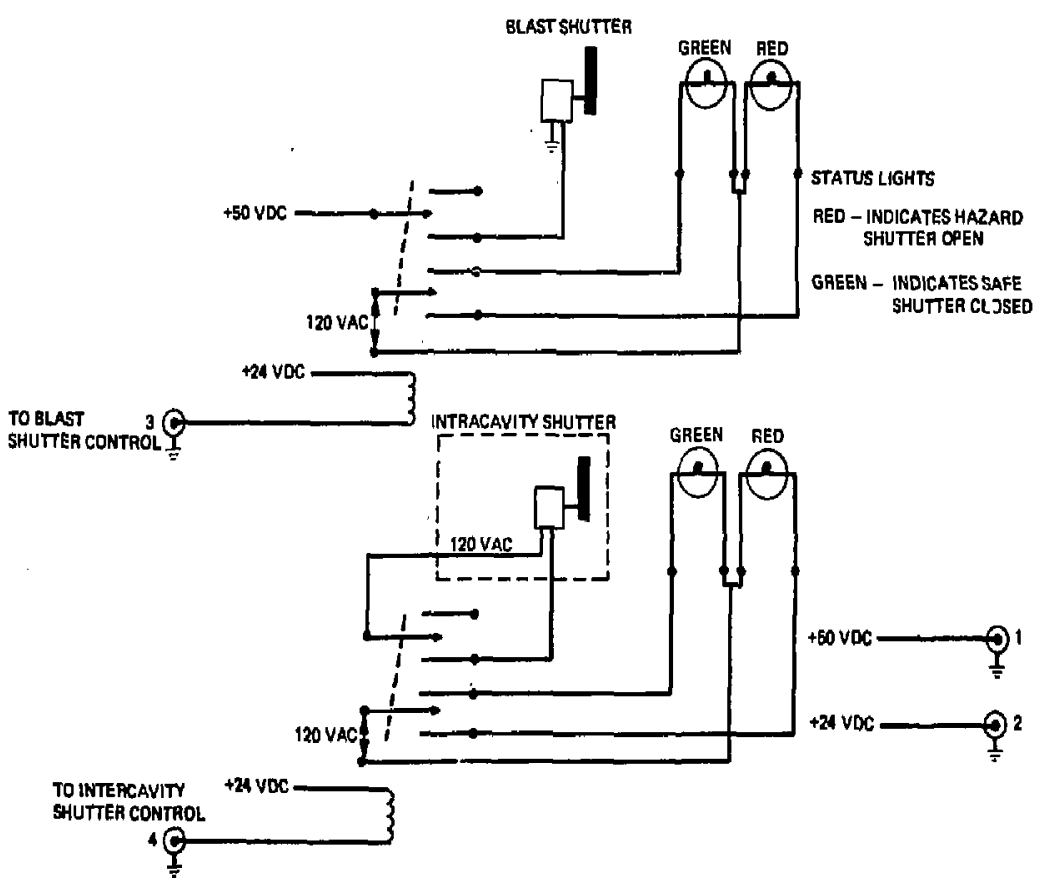

Figure 4-10. Electronics - optical table - shutter systems box. 


\subsection{DESCRIPTION - CHOPPER REFERENCE SIGNAL CIRCUIT (FIGURE 4-11)}

The chopper motor is a synchronous 3600 RPM AC motor. It drives a $\mathrm{Zl}$ opening dual blade. The two blades are necessary to precisely adjust the required 50 percent duty cycle. A 1-2.29 drive pulley causes the blade to chop at:

$$
\frac{3600}{60} \times 21 \times \frac{1}{2.29}=550 \mathrm{~Hz}
$$

A reference for the PAR lockin amplifier is developed by optical pickup (LED-Phototransistor). The signal waveform is processed by a Schmidt trigger and a $50 \mathrm{ohm}$ line driver to drive the long coaxial interconnection.

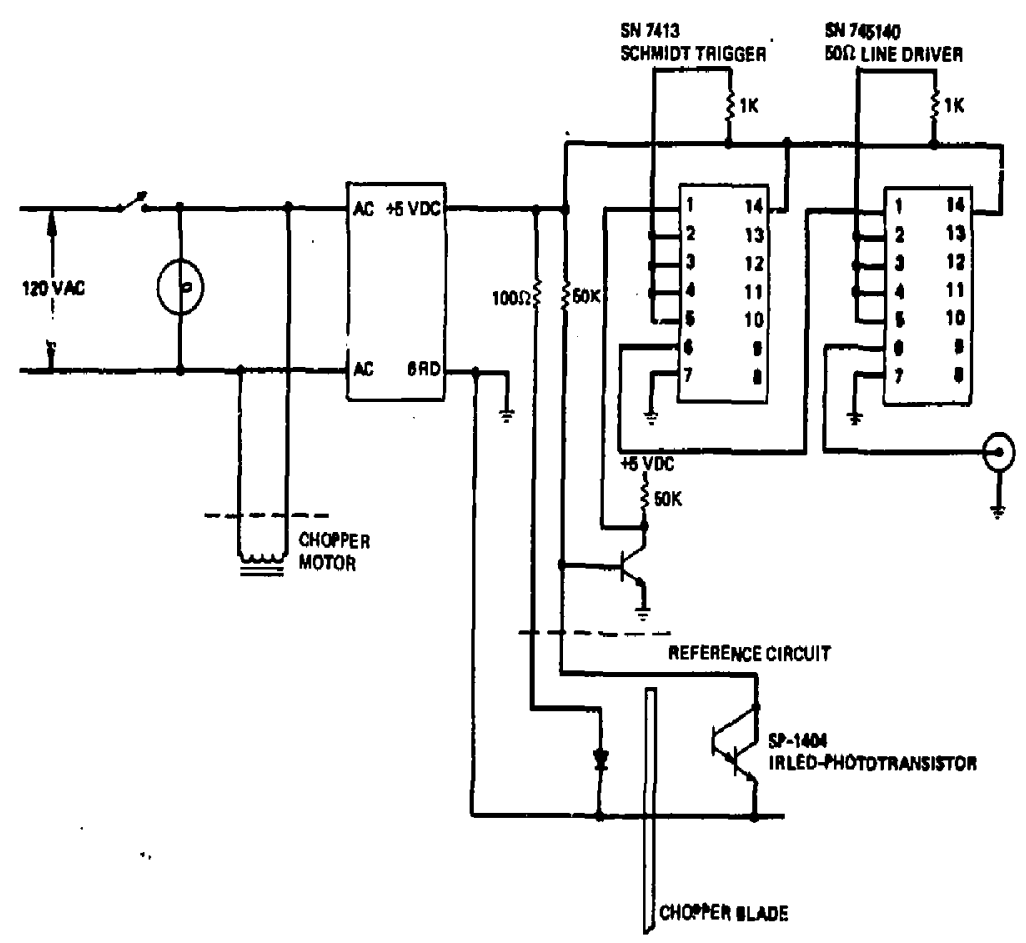

Figure 4-11, Electronics - optical table - chopper reference signal circuit. 


\section{12 DESCRIPTION - LASER HOOKUP AND OPERATION (FIGURE 4-12)}

The Quantronixs laser is mounted on the optics table, separated irom its lamp supply, closed loop water flow, and heat exchanger. The following lists required connections.

1. A five wire cable brings in 20830 at 40 amps/phase using 5 wires to the back of the lamp supply. There are three (3) phases, a neutral, and a chassis ground.

2. An AC interconnection cable goes from the lamp sipply to the lower cabinet position to the pump, and electronics. Once this cable is in place, a "correct" phase rotation indicator light is operative and must be illuminated for proper pump rotation.

3. A large two wire HV encapsulated goes from the supply to the table for the lamp.

4. A smaller cable for the thermal and flow sensors also goes to the table.

5. Two water tubes, supply and return also go to the table.

6. A 120 VAC standard rip cord goes to the optics table.

7. Water lines from an external supply goes into the lower cabinet flowing through the heat exchanger.

From the modelocter driver

1. 120 VAC input

2. Sync output to sampling plugin

3. AO drive coax (maintain lengths) goes to an inline power meter, and on to the table.

From the electronics cabinet

1. 10 separate miniature coax cables mate with the connectors on the table.

2. 120 VAC input PAR

3. 120 VAC input electronics

The system is provided with various interlocks which must be closed for operation. An interlock on the lasir cover opens when the cover is removed. A removable cap is attached to an interlock at the rear mirror, to vrevent operation with rear output. Since the output is used for modelock pulse monitoring it is removed from the cap. 


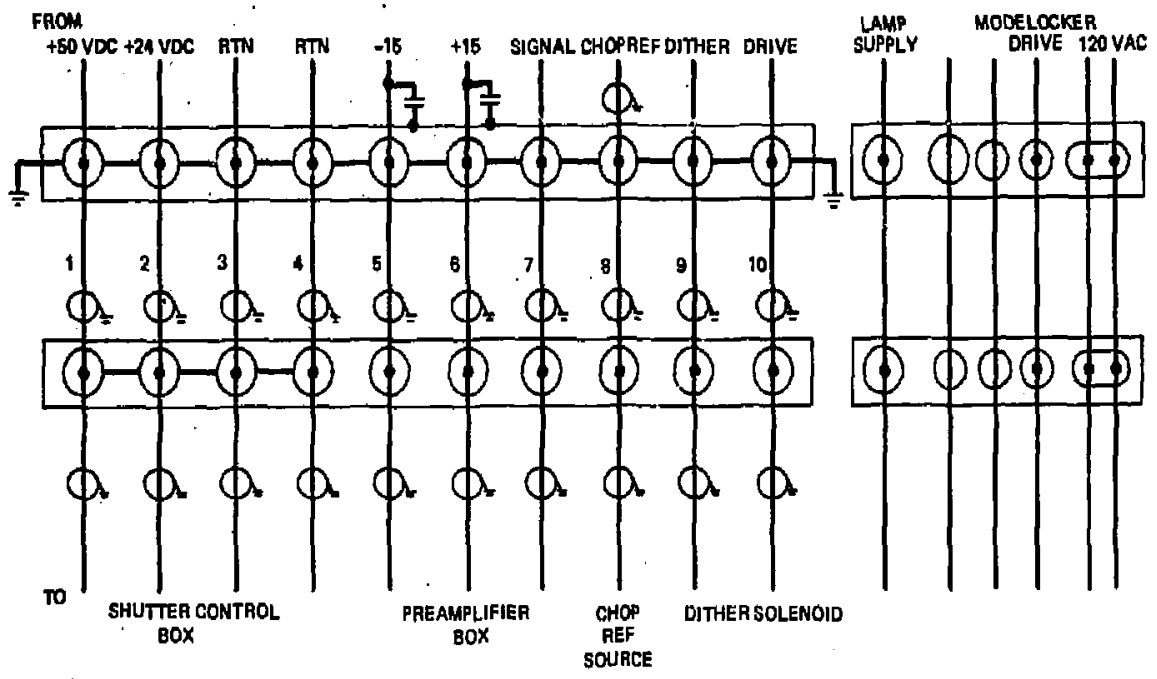

a. Electronics - Optical Table - Electronics Hookup
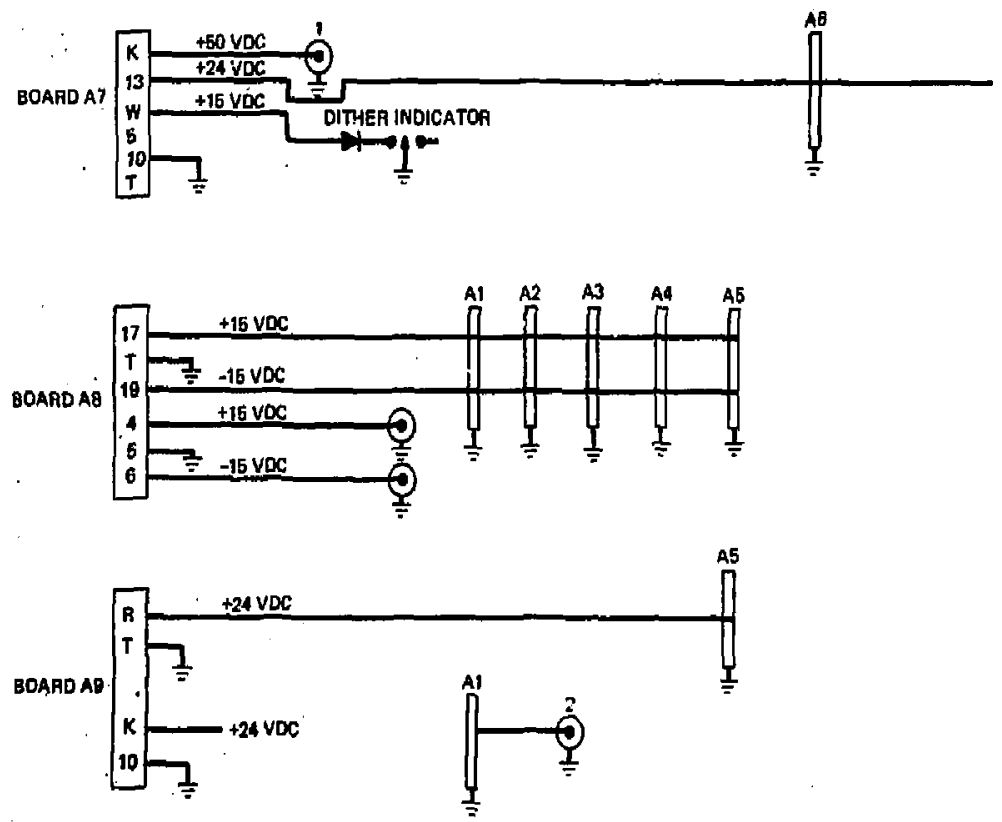

b. Electronics - Power Distribution Diagram

Figure 4-12. Laser hookup and operation.

44 
The laser is turned on by

1. Turn on Key
a. Power Indicator will Light
b. Interlock Indicator Must Not Light
c. Level Indicat's Must Not Light

2. Depress Pump

3. Turn on External Cooling Water to Heat Exchanger

4. Set Lamp Level to Half Scale, Depress Switch

5. When Lamp Lights, Set Level to 20.0 amps

6. Leave on Modelocker Driver at All Times. 


\subsection{DESCRIPTION - DETECTORS AND PREAMPLIFIER (FIGURE 4-13)}

The detectors are biased and their output currents amplified by a transimpedance ar.aplifier. The current node performs the differencing for the two curres.ts required Ly the balanced detection process. The LH0032 is a high input impedance precision operational amplifier. The output drives a buffer amp and zoaxial line driver. This buffer is required to drive the long coax cable return to the receiver.

In add tion this buffer provide the isolation of the preamp from driving the large coarial capacitance, required for stability.

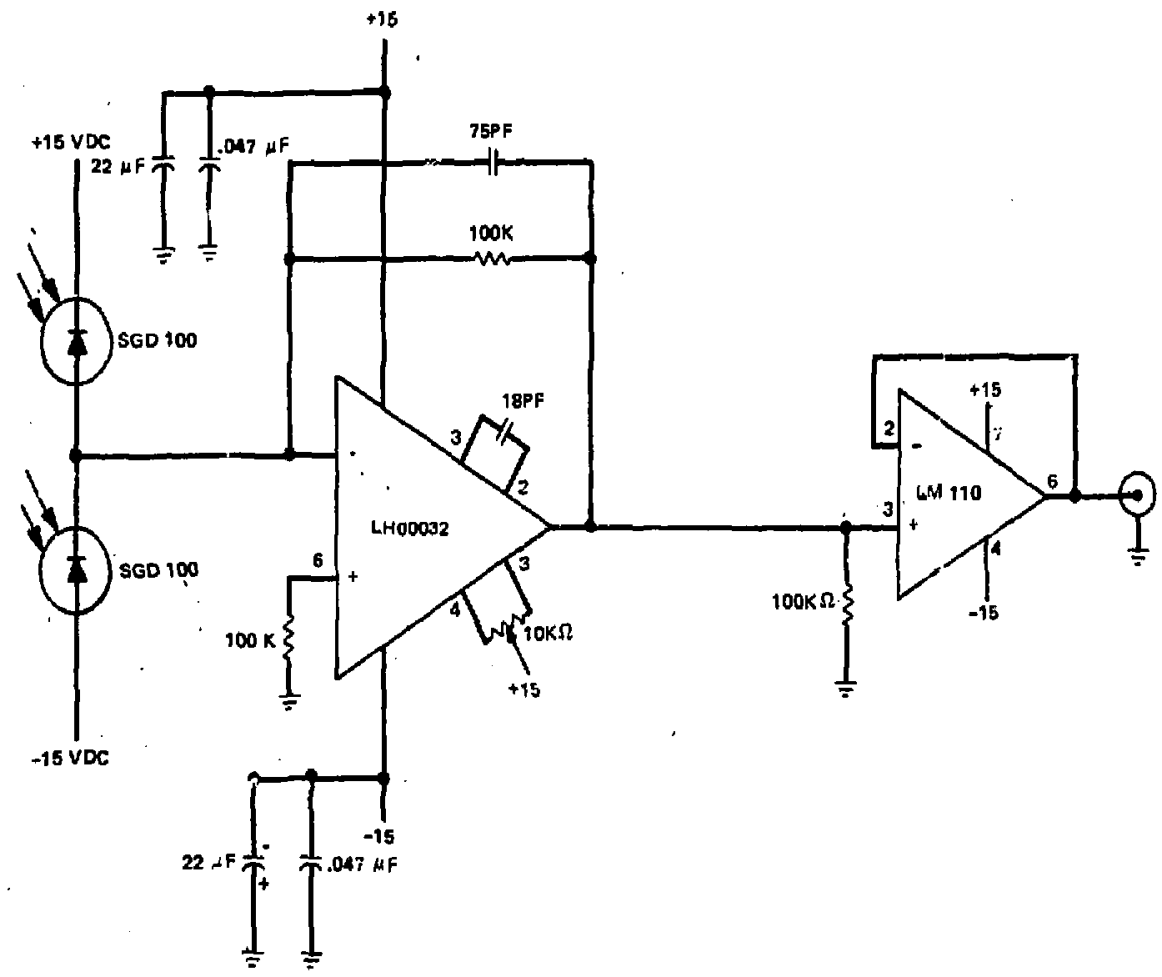

Figure 4-23. Electronics - Optical Table - Detectors and Preamplifier. 
4.14 ELECTRONICS CONTROL PANEL (FIGLRE 4-14)

\begin{tabular}{|c|c|c|c|c|c|c|c|c|}
\hline \multirow{2}{*}{ Junction } & \multicolumn{4}{|c|}{ From } & \multicolumn{4}{|c|}{ Io } \\
\hline & Board & $\operatorname{Pin}$ & Osm No. & Eloment No. & Board & Pin & Osm No. & Element No. \\
\hline 1 & $A^{8}$ & 17 & & & & & & \\
\hline 2 & & GRD & - & & & & & \\
\hline 3 & A8 & 19 & & & & & & \\
\hline 4 & A9 & $\mathbf{R}$ & & & & & & \\
\hline 5 & & GRD & & & & & & \\
\hline 6 & Aล & 4 & & & & & & \\
\hline 7 & & GRD & & & & & & \\
\hline 8 & $A B$ & 6 & & & & & & \\
\hline 9 & A9 & $\mathrm{k}$ & & & & & & \\
\hline 10 & & GRD & & & & & & \\
\hline $1 !$ & & & & 13 Switch & & & & 17 \\
\hline 12 & & & & $\mathrm{~N} / \mathrm{C}$ & & & & \\
\hline 13 & & & & 16 & & & & II Switch \\
\hline 14 & & & & 11 & A7, A8, A9 & $A-\%$ & & \\
\hline 15 & $120 \mathrm{VAC}$ & IN_COLD & & & A7, AB, A9 & $A=\%$ & & \\
\hline 16 & & & & 17 Fuse & & & & 13 \\
\hline 17 & $120 \mathrm{VAC}$ & IN-HOT & & & & & & 16 Fuse \\
\hline 18 & & & & 22 & & & & Exterras Short \\
\hline 19 & & & & LI Switch & AlV & & & \\
\hline 20 & & & & $N E$ & & & & \\
\hline 21 & & & & 23 & & & & 19 Switch \\
\hline$\measuredangle 6$ & 4. & $\mathbf{P}$ & & & & & & 23 LED \\
\hline 23 & & & & 22 1.ED & & & & 21 \\
\hline 24 & & & & 26 Swilch & A) $1:$ & & & \\
\hline 25 & & & & $\mathrm{~N} / \mathrm{C}$ & & & & \\
\hline 26 & & & & 28 & & & & 24 5witch \\
\hline 27 & Al & s & & & & & & 28 LED \\
\hline 28 & & & & 27 LED & & & & 26 \\
\hline 29 & & & & 27 & & & & External Short \\
\hline 30 & & & & 32 Switch & & & & 34 \\
\hline 31 & & & & 32 Switch & & & & 37 \\
\hline 32 & & GRD & & & & & & 30, 31 Switch \\
\hline 13 & A7 & w & & & & & & $3+$ LED \\
\hline 34 & & & & 30 & & & & 33 LED \\
\hline
\end{tabular}




\section{14 ELECTRONICS CONTROL PANEL (Continued)}

\begin{tabular}{|c|c|c|c|c|c|c|c|c|}
\hline \multirow[b]{2}{*}{ Junction } & \multicolumn{4}{|c|}{ From } & \multicolumn{4}{|c|}{ To } \\
\hline & Board & Pin & Osm No. & Element No. & Board & Pin & Osin No. & External No. \\
\hline 35 & A5 & $\mathrm{k}$ & & & & & & 36, 37 Pot \\
\hline 36 & & GRD & & & & & & 35, 37 Pot \\
\hline 37 & & & & 35 Pot & A5 A & & . & \\
\hline 38 & A5 & $M^{\prime}$ & & & & & & Dither Ref Monitor \\
\hline 39 & $\mathrm{~A}^{3}$ & $\mathrm{u}$ & & . & & & & 40 Pot \\
\hline 40 & A3 & $\mathrm{v}$ & & & & & & $39 \mathrm{Pot}$ \\
\hline 41 & A4 & $\mathrm{K}$ & & & A3 A & & & 42 \\
\hline$\$ 2$ & & & Nn. 7 & & A4 $\mathrm{A}$ & & & \\
\hline 43 & & & No. 8 & $\cdot$ & & & & Chopper Reference \\
\hline 44 & A2 & $\mathrm{k}$ & & - & & & & 45,46 Pot \\
\hline 45 & 93 & & & 44,46 Pot & & & & \\
\hline 46 & & & & $\$ 4$ Pot & A2 A & & & \\
\hline 47 & $A^{j} \mathfrak{s}$ & $\mathbf{P}$ & & & & & & Boost Amp Monitor \\
\hline 48 & $\mathrm{~A} 2$ & K & & & & & & Luckin Input \\
\hline 49 & A2 & $M$ & & & & & & $50 \mathrm{Pot}$ \\
\hline so & & & & 49 Pot & A2 D & & & \\
\hline 51 & A2 & $\mathrm{x}$ & & & & . & & 53 Pot \\
\hline 52 & $\mathrm{~A}^{2}$ & $w$ & ' & & & & & 53 Pot \\
\hline 53 & & & & 51, 52 Pot & A2 Y Y & & & \\
\hline 54 & A3 & $\mathrm{k}$ & & & & & & Envelope Det Monitor \\
\hline
\end{tabular}




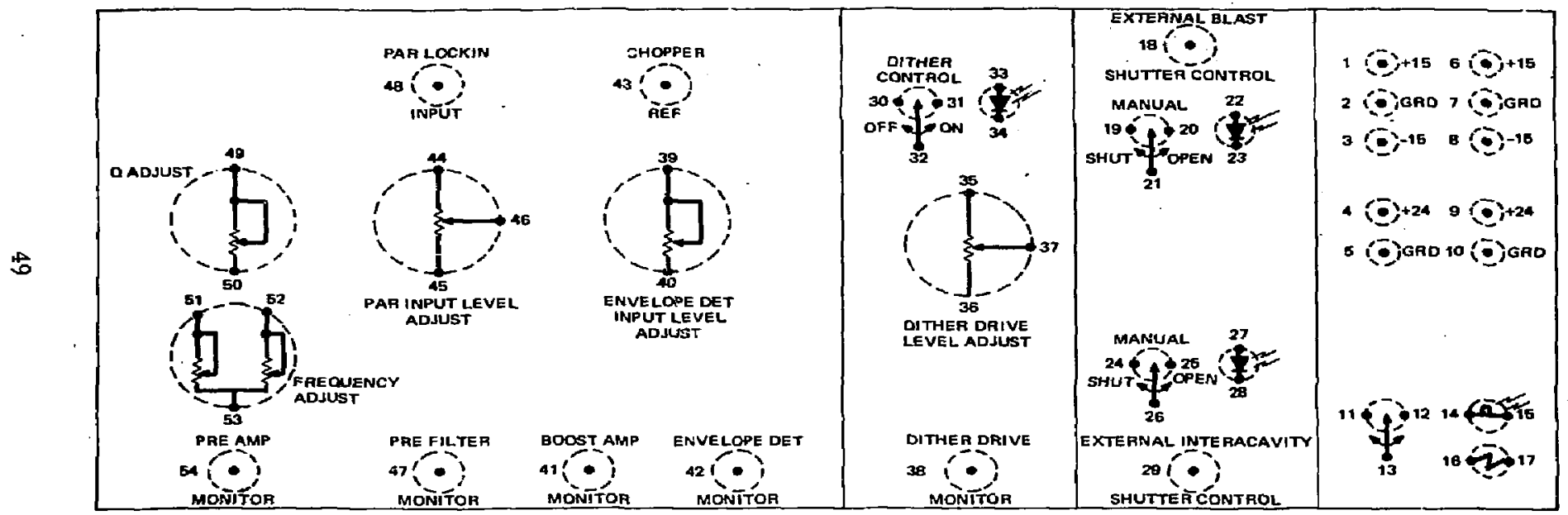

Figure 4-14. Electronics control panel. 


\section{0 SUPPORTING CALCULATIONS}

\section{I CHARACTERIZATION OF THE SIGNALS}

The time dependence of E field output of any CW laser may be written

$$
E(t)=\sum_{n} E_{n} e^{i \omega_{n} t+i \varphi_{n}}
$$

where the summation is over several modes of frequency $\omega_{n}=\omega_{0}+2 \pi n / T$ and positive, real amplitudes $E_{n}=E^{0} Y_{n}$, where $Y_{n} \approx \exp \left(-a_{n}^{2}\right)$ describes the mode amplitude distribution. $E(t)$ is periodic in $T=2 \mathrm{~L} / \mathrm{C}$, where $\mathrm{L}$ is the laser cavity optical length. When the laser is mode locked, the number of mortes in oscillation generally increases, and all of the modes add in phase at some time which may be arbitrarily set equal to zero. All $\varphi_{n}$ may then be set equal to zero.

The detector response when irradiated by the signal $\mathrm{E}_{\mathbf{s}}$ and local oscillator $E_{L O}$ is

$$
R(\tau)=S\left|E_{S}+E_{L O}\right|^{2}
$$

where

$$
\begin{aligned}
S & =\text { detector responsivity }=\eta \text { e/hv amp/watt } \\
T & =\text { time delay between } E_{S} \text { and } E_{L O} \\
E_{B} & : E_{S}^{0} \sum_{n} Y_{n} e^{i \omega_{n}(t+T)}
\end{aligned}
$$




$$
\begin{aligned}
& E_{L O}=E_{L O}^{0} \operatorname{Re}\left|\sum_{n} \gamma_{n} e^{i\left(\omega_{n}+\omega_{L O}\right) t}\right|=E_{L O}^{0} \sum_{n} \gamma_{n} e^{-i \omega_{n} t} \cos \omega_{L O} t \\
& \omega_{L O}=\text { LO frequency offset. }
\end{aligned}
$$

Then the evaluation of $(5-2)$ for the amplitude of the term at frequency $w_{\text {LO }} \ll w_{n}$ is

$$
\begin{aligned}
I_{g}(\tau) & =2 S E_{L O}^{0} E_{S}^{0} \sum_{n} \gamma_{n} e^{i \omega_{n}(t+\tau)} \sum_{m} \gamma_{m} e^{-i \omega_{m} t} \\
& =2 S E_{L O}^{0} E_{S}^{0} \sum_{n} \gamma_{n}^{2} e^{i \omega_{n} T}
\end{aligned}
$$

for $n=m$, since the IF bandpass excludes terms with $n \neq m$. From (5-4), the peak current occurs when $\tau=0$, or

$$
I_{s}\left(\text { peak) }=I_{s}(0)=2 S E_{L} \circ E_{S}^{0} \sum_{n} \gamma_{n}^{2}\right.
$$

The dependence of $I_{g}$ (peak) on laser power will now be calculated. The signal power is given by

$$
\begin{aligned}
P_{S}(t) & =E_{S}(t) E_{S}^{* k}(t) \\
& =\left(E_{S}^{0}\right)^{2} \sum_{n} \sum_{m} Y_{n} Y_{m} e^{i\left(\omega_{n}-\omega_{m}\right) t}
\end{aligned}
$$


The average power is

$$
\left\langle P_{s}(t)\right\rangle=\frac{1}{T} \int_{0}^{T} d t P_{s}(t)=\left(E_{s}^{0}\right)^{2} \sum Y_{n}{ }^{2},
$$

and the peak power is

$$
P_{s}(\text { peak })=P_{s}(0)=E_{s}^{2}\left(\sum_{n} y_{n}\right)^{2}
$$

Then from $(5-5)$ and $(5-6)$,

$$
I_{g} \text { (peak) }=25<\mathrm{PO}_{L O^{1 / 2}}\left\langle\mathrm{P}_{8}\right\rangle^{1 / 2} \text {, }
$$

and it is concluded that the SNR calculations to follow depend on average powers rather than peak power. 


\subsection{THE CARRIER SIGNAL TO NOISE RATIO (SNR ${ }_{c}$ )}

The rms shot noise at the output of a silicon diode is

$$
\left(4 \text { e I } I_{d c} B_{I F}\right)^{1 / 2} \text {, }
$$

where

$$
\begin{aligned}
I_{d c} & =S\left\langle P_{L O}\right\rangle=\text { direct current flow } \\
e & =\text { electron charge }=1.6 \times 10^{-19} \text { coul } \\
B_{\text {IF }} & =I F \text { bandpass, } \mathrm{HZ} .
\end{aligned}
$$

Then the IF zero-to-peak to rms noise is

$$
\begin{aligned}
\operatorname{SNR}_{c}(\tau) & =\frac{I_{s}(\tau)}{\left(4 e S<P_{L O}>B_{I F}\right)^{1 / 2}} \\
& =\left(\frac{\eta<P_{S}>}{h v B_{I F}}\right)^{1 / 2}\left(\frac{I_{g}(\tau)}{I_{g}(0)}\right),
\end{aligned}
$$

where from $(5-4)$,

$$
\begin{aligned}
\frac{I_{s}(\tau)}{I_{s}(0)}= & \frac{\sum_{n} \gamma_{n}{ }^{2} e^{i \omega_{n} \tau}}{\sum_{n} \gamma_{n}{ }^{2}} \\
& =\frac{\sum e^{-2 a n^{2}+i \omega_{n} \tau}}{\sum e^{-2 a n^{2}}}
\end{aligned}
$$


Typically, 20 to 50 modes are oscillating so that the summations in (5-10) can be approximaled by integrals:

$$
\begin{aligned}
I_{s}(T) \sim \sum_{-\infty}^{\infty} e^{-\tan ^{2}+i 2 \pi n \frac{I}{T}} & \sum_{-\infty}^{\infty} e^{-2 a n^{2} \cos \left(2 \pi n \frac{T}{T}\right)} \\
& \approx \int_{-\infty}^{\infty} e^{-x^{2}} \cos \left[\frac{2 \pi}{\sqrt{2 a}}\left(\frac{T}{T}\right) x\right] \frac{d x}{\sqrt{2 a}} \\
& =\sqrt{\frac{\pi}{2 a}} e^{-\frac{1}{2 a}\left(\frac{I T}{T}\right)^{2}}
\end{aligned}
$$

The validity for replacing $(5-11)$ by $(5-12)$ is seen to depend on the value of $T / T ;$ if $T / T>1 / 12$, then argument of the cosine changes by greater than $30^{\circ}$ for each increment of $\mathrm{n}$ by one and the continuous approximation is greatly in error. Therelore, $(5-13)$ is good only near the peak at $\tau=0$. However, if a is small (large number of modes oscillating) then (5-13) is narrow and $(5-13)$ will be valid where $I(\tau)$ is large. For example, if $N$ modes are oscillating, $a \approx 4 \mathrm{~N}^{-2}$, and

$$
\frac{I_{s}(\tau)}{I_{s}(0)}=e^{-\frac{1}{8}\left(\frac{N \pi \tau}{T}\right)^{2}}=e^{-1}
$$

for

$$
\left(\frac{\tau}{T}\right)_{e-1}=\frac{2 \sqrt{2}}{\pi N} \approx \frac{1}{N} .
$$

so that this approximation for $T / T$ is adequate provided $N>12$. 
It is of interest to compare the width of $I_{S}(T)$ from $(5-15)$ with the pulse width of $P_{s}(t)$ :

$$
\begin{aligned}
\frac{P_{s}(t)}{P_{s}(0)} & =\left|\frac{\sum_{n} \gamma_{n} e^{i \omega_{n} t}}{\sum_{n} Y_{n}}\right|^{2} \\
& {\left[e^{\left.-\frac{1}{a}\left(\frac{\pi t}{T}\right)^{2}\right]^{2} \text { (from 5-13 with 2a - a) }}\right.} \\
= & e^{-\frac{2}{a}\left(\frac{\pi t}{T}\right)^{2},}
\end{aligned}
$$

and

$$
\left(\frac{t}{T}\right)_{e^{-1}}=\frac{1}{\pi} \sqrt{\frac{a}{2}}=\frac{\sqrt{2}}{\pi N}=\frac{1}{2}\left(\frac{T}{T}\right)_{e^{-1}} .
$$

The width of the response curve $I_{s}(T) / I_{s}(0)$ is therefore twice that of the mode locked pulse width. 


\section{3 THE DOUBLE LO TECHNIQUE FOR POSITION MEASUREMENT}

As shown in the previous section, the IF current varies approximately as a gaussian with the delay time $T$, which may be related to the difference in optical path lengths of the LO and signai arms (neglecting multiples of $1 / 2$ the cavity length):

$$
X=\frac{c \tau}{2} \text {. }
$$

In terms of $\mathrm{X}$,

$$
f(x) \equiv \frac{I_{s}(\tau)}{I_{s}(0)}=e^{-(X / \sigma)^{2}}
$$

where

$$
\sigma=\frac{\sqrt{2}}{\pi} \frac{c T}{N}
$$

When two LO paths are alternately presented to the detector by means of a chopper, the peak-to-peak modulated signal current (square wave) becomes

$$
I_{m}(z) \sim Q f\left(X-\frac{\Delta}{2}\right)-f\left(X+\frac{\Delta}{2}\right)
$$

where $\Delta$ is the optical path length separation of the two $L O$ paths and $Q$ is the amplitude mismatch of the two LO beams. The equalization of the path lengths consists of adjusting $X$ for each of the amplifier arms so that $I_{m}=0$ with a fixed LO configuration. $(5-20)$ is plotted in Figure 5-1 for several values of $\Delta / 2 \sigma$ and for $Q=1$. 


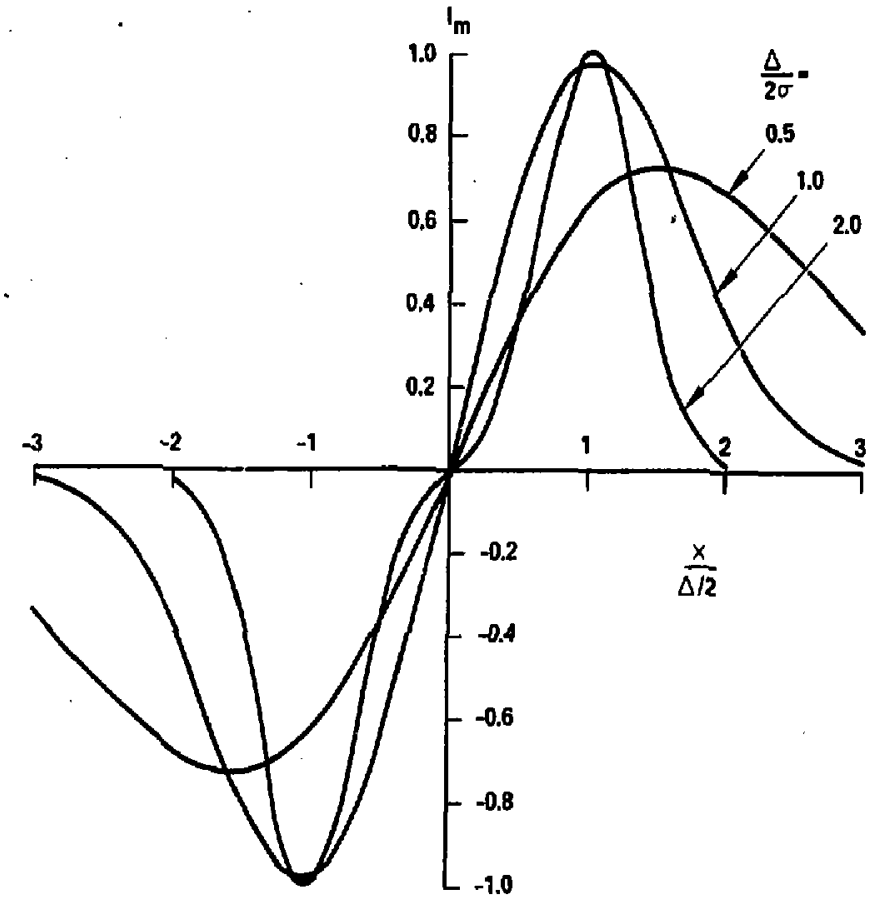

MAY 1977

Figure 5-1. Variation of signal current with path length difference.

For small errors in $\mathrm{X}$ away from $\mathrm{X}=0$,

$$
\begin{aligned}
I_{m}(\delta X) & =I_{m}(0)+\delta X I_{m}^{\prime}(0) \\
& =0+\delta x\left[\frac{2 \Delta}{\sigma^{2}} e^{-\left(\frac{\Delta}{2 \sigma}\right)^{2}}\right],
\end{aligned}
$$


and the sensitivity to $\delta \mathrm{X}$ is described by

$$
\begin{aligned}
\operatorname{SNR}_{\mathrm{m}}(\delta \mathrm{X}) & =\left(\frac{\eta\left\langle\mathrm{P}_{\mathrm{g}}\right\rangle}{h v \mathrm{~B}_{I F}}\right)^{1 / 2}\left(\tau_{i} B_{I F}\right)^{1 / 2} I_{m}(\delta \mathrm{X}), \\
& =\left(\frac{\delta \mathrm{X}}{\sigma}\right) F_{1}\left(\frac{\Delta}{2 \sigma}\right)\left[\frac{\eta\left\langle\mathrm{P}_{E}\right\rangle r_{i}}{h_{v}}\right]^{1 / 2}
\end{aligned}
$$

where

$$
\begin{aligned}
\tau_{\mathfrak{i}} & =\text { integration time of the phase lock amplifier. } \\
F_{1}(X) & \equiv 4 \mathrm{Xe}^{-\mathrm{x}^{2}} .
\end{aligned}
$$

Equation (5-23) is appropriate for envelope detection of an IF carrier with SNR $_{I F} \gg 1$, and subsequent synchronous detection of the AM modulation with integration time $T_{\mathfrak{i}}$.

The function $F_{1}(X)$ is plotted in Figure $5-2$ and exhibits a maximum at $\mathrm{X}=1 / \sqrt{2}$. The optimum optical pathlength difference $\Delta$ is therefore

$$
\Delta=\sqrt{2} \sigma
$$

at which point

$$
\operatorname{SNR}_{m}(\delta X)=(1.7) \frac{\delta X}{\sigma}\left[\frac{\eta\left\langle P_{s}\right\rangle r_{i}}{h v}\right]^{1 / 2} .
$$

Finally, an imbalance $Q$ in the two LO powers does not effect the measurement provided the imbalance is not too large; the null point of $I_{m}(x)$ in $(5-20)$ is $X=X_{0} \neq 0$ and $X_{0}$ is the same for all amplifier channels because it is independent of the returned signal power. 


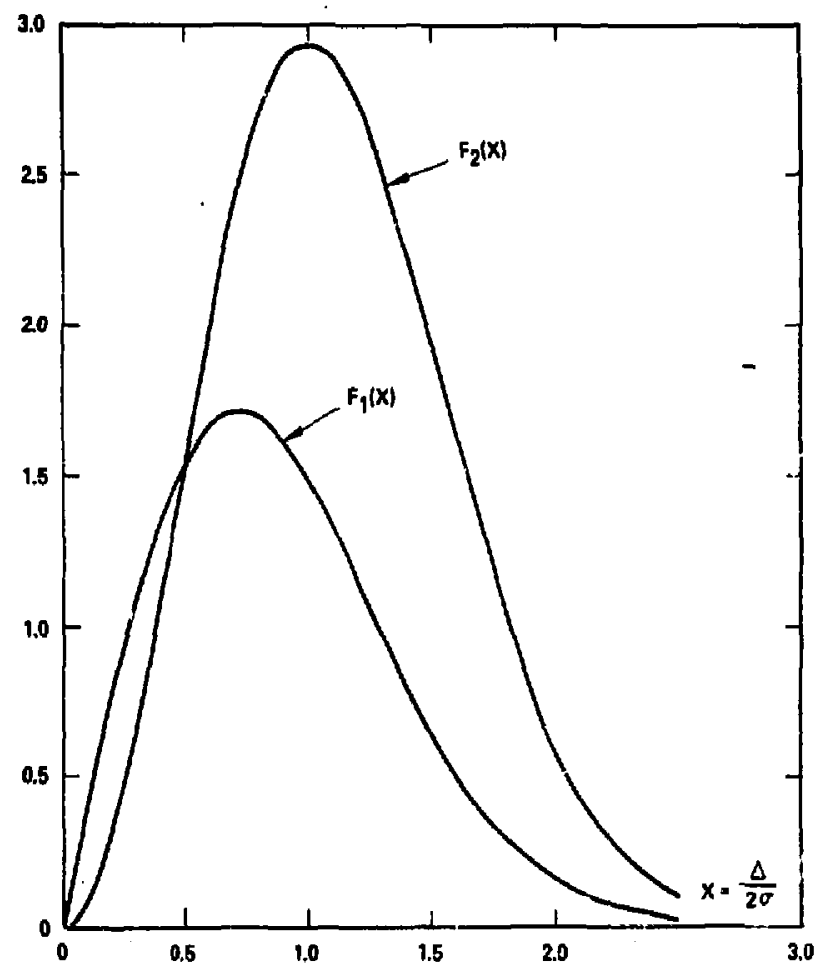

MAY 1977

Figure 5-2. Dependence of SNR on the ratio $\Delta / 2 \sigma$. 


\section{4 LO BEAMSPLITTER ANALYSIS}

Two opecially fabricated beamsplitters provide the two LO optical pathlengthe required for the system. In Figure 5-3, the two paths are

1. $A B$

2. ACDEF'

and they are alternately obscured by a chopper with blade spacinge fabricated to match the opacing beams (1) and (2). The optical path difference between (1) and (2) is given by

$$
\Delta=4 \mathrm{t}\left\langle\mathrm{n}^{2}-\sin ^{2} \theta\right\rangle^{1 / 2}:=6.44 \mathrm{~cm}
$$

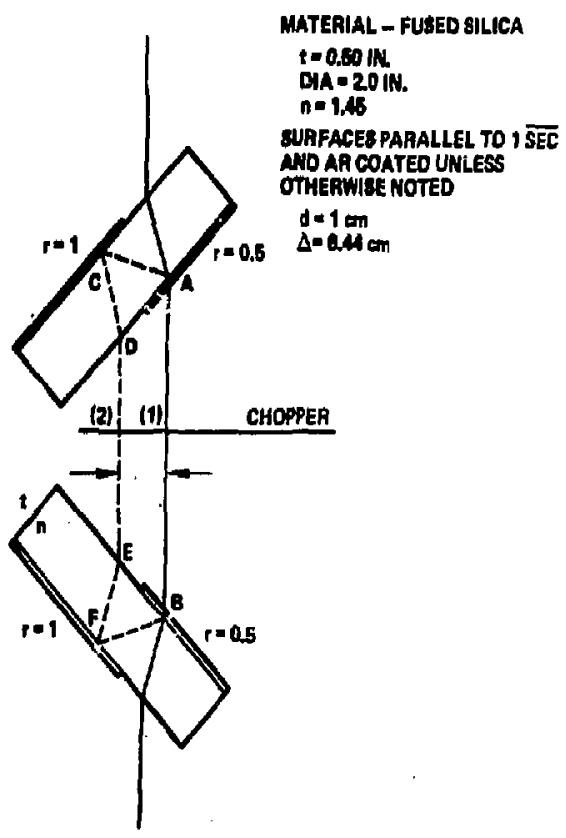

Eigure 5-3. LO beamsplitter configuration. 
and the beam epacing by

$$
d=\frac{t \sin 2 \theta}{\left(n^{2}-\sin ^{2} \theta\right)^{1 / 2}}=1.00 \mathrm{~cm}
$$

for $\theta=45^{\circ}, \eta=1.45$, and $t=0.5 \mathrm{in}$. From $(5-16),(5-17)$, and $(5-24), \Delta$ is the optimum pathlength difference for the following pulsewidth:

$$
(2 t)_{e^{-1}}=\frac{1}{2}(2 \mathrm{~T})_{\mathrm{e}^{-1}}=\frac{1}{2} \frac{2}{\mathrm{C}}(2 \sigma)=\frac{\sqrt{2}}{\mathrm{C}} \Delta=300 \mathrm{psec}
$$

It is of interest to examine the variation of SNR $_{m}$ with the laser pulse width $2 / \mathrm{c}$, keeping $\Delta$ fixed. Equation $(5-23)$ is rewritten

$$
\operatorname{SNR}_{m}(\delta X)=\left(\frac{\delta X}{\Delta}\right) F_{2}\left(\frac{\Delta}{2 \sigma}\right)\left(\frac{\eta<P_{s}>T_{i}}{h v}\right)^{1 / 2}
$$

where

$$
F_{2}(X)=8 x^{2} e^{-x^{2}}
$$

is plotted in Figure, $(5-1)$. If now $\Delta$ is fixed at $6.44 \mathrm{~cm}$, the curve for $F_{2}$ shows that a pulse width $\left(e^{-1}\right.$ points)

$$
\frac{2 \sigma}{\mathrm{C}}=\frac{\Delta}{\mathrm{C}}=215 . \mathrm{psec}
$$

is optimum, and that the loss in $S N R{ }_{m}$ is less than a factor of two if the pulse width falls between 134. and 430. psec.

It is finally noted that Figure $5-2$ is simplified in that it does not consider polarization. When employed in the system, the polarization is p-wave on the first pass through the beamsplitters. The cat's eye and quarter 
wave plate rotate the polarization by $90^{\circ}$ so that S-wave polarization traverses the beamsplitters on the return pass. If the losses at the antireflection coated surfaces are neglected then the ratio of $\mathrm{LO}_{1}$ to $\mathrm{LO}_{2}$ power $\mathrm{R}$ is given by

$$
R^{1 / 2}=\frac{r_{s} r_{p}}{t_{s} t_{p}}=\frac{\left(1-t_{s}\right)\left(1-t_{p}\right)}{t_{s} t_{p}}
$$

where

$$
\begin{aligned}
& t_{s}\left(r_{s}\right)=s \text {-wave transmission (reflectance) } \\
& t_{p}\left(r_{p}\right)=p \text {-wave transmission (reflectance). }
\end{aligned}
$$

If $t_{s}+t_{p}=1$, then $R=1$. This is the correct condition on the beamsplitter coating, replacing $R=t=0.5$. 


\subsection{EFFECTS OF ABERRATIONS ON SNR}

The aberrations in the return signal wavefront are unknown, but are expected to be on the order of one wave. A calculation of the signal loss incurred by 1) a wavefront tilt and 2) spherical aberration is given below for a gaussian intensity profile.

The heterodyned signal is proportional to an integral over the reciver aperture:

$$
\operatorname{Re} \int \underline{d r} E_{s}(\underline{r}) E_{L O}^{*}(\underline{r})
$$

where

$$
\begin{aligned}
E_{\theta}(\underline{r}) & =\text { return signal wavefront }-\sqrt{P_{s}} e^{i \phi-r^{2} / w^{2}} \\
E_{L O}(\underline{r}) & =\text { local oscillator wavefront }-\sqrt{P_{L O}} e^{-r^{2} / w^{2}} \\
\phi & =\text { signal wavefront aberration. }
\end{aligned}
$$

The receiver noise is proportional to $\sqrt{\mathrm{P}_{\mathrm{LO}}}$, so that the IF peak-to-rms noise ratio $\left(\mathrm{SNR}_{I F}\right)$ is proportional to

$$
\mathrm{SNR}_{I F} \sim \sqrt{\mathrm{P}_{\mathrm{s}}} \operatorname{Re} \iint \mathrm{rdrd} \theta \mathrm{e}^{i \phi-2 \mathrm{r}^{2} / \mathrm{w}^{2}}
$$

\section{Wavefront Tilt}

In this case, $\phi=\beta r \cos \theta$. If we define a full wave aberration such that $\phi=\pi$ for $r=w$, then $\beta=\pi / w$. The integral becomes

$$
I=R e \int_{0}^{\infty} r d r \int_{0}^{2 \pi} d \theta e^{i \beta r \cos \theta-2 r^{2} / w^{2}}
$$




$$
\begin{aligned}
& -\int_{0} \operatorname{rdr} e^{-2 r^{2} / w^{2}} J_{0}(\beta r) \\
& -\exp \left[-\beta^{2} w^{2} / 8\right] \begin{array}{l}
\text { (Gradshteyn and Ryzhik } \\
\text { p. } 717, \text { No. } 7)
\end{array}
\end{aligned}
$$

where only the dependence on the wave tilt $\beta$ has been retained, and the integral over $r$ has been extended to infinity. For $\beta=\pi / w$, the reduction in SNR due to tilt is

$$
\exp \left[-\pi^{2} / 8\right]=0.29
$$

\section{Spherical Aberration}

In this case, $\phi=\alpha s^{2}$, and

$$
\begin{aligned}
& I-\operatorname{Re} \int_{0}^{\infty} r d r \exp \left[i \alpha r^{2}-2 r^{2} / w^{2}\right] \\
& -\operatorname{Re} \frac{1}{\left(i \alpha-2 / w^{2}\right)} \\
& =\frac{1}{1+\left(\alpha w^{2} / 2\right)^{2}}
\end{aligned}
$$

For $\phi=2 \pi$ when $r=w$, obtain $\alpha=2 \pi / w^{2}$, and the SNR reduction factor is

$$
\frac{1}{1+\pi}=0.09
$$




\section{Discussion}

An alternate approach is to expand the signal field into a series of or thogonal modes. The number of modes required in the expansion is limited by the pin hole size in the spatial filter. A single mode LO beam selects out one of these signal modes, whose coefficient determines the heterodyned signal. A multimode LO might achieve a higher SNR, depending on the relative phases between modes. A complete analysis along these lines was not felt to be warranted.

The beam profile intensity may actually be "super" gaussian (I $\left.\sim \exp \left[-r^{n}\right], r>2\right)$, on which case the above results would become more complicated mathematically, but perhaps not qualitatively so, as $\eta \rightarrow \infty$, we return to the uniform beam profile, which exhibits nulls for several values of $\alpha$ and $\beta$. Whether these nulls occur for $\eta=5$ has not been examined. In any event, the $\eta=2$ case is convenient because one need not worry about finding himself in a null when a signal is present, or of aligning the receiver against a secondary maximum. 


\subsection{GHOST SIGNALS}

Optical surfaces in the amplifier chain might produce reflections which could dominate the highly attenuated $\left(10^{-9}\right)$ return from the surrogate target. Considering first specular. reflections, the situation is illustrated in Figure 5-4. A beam with radius of curvature $R_{1}$ is incident on surface $S$ with radius $R_{s}$. The transformation of a gaussian beam at an interface $S$ may be described by the $A B C D$ formalism *:

$$
\begin{aligned}
& \frac{1}{q}=\frac{1}{R}-\frac{i 2}{k_{0} w^{2}} \\
& k_{0}=2 \pi / \lambda_{0}\left(\lambda_{0}=\right.\text { wavelength in a vacuum) } \\
& n=\text { optical index } \\
& R=\text { radius of curvature } \\
& \omega=\text { spot radius (E-field } e^{-1} \text { radius) } \\
& q_{2}=\frac{A q_{1}+B}{C q_{1}+D} .
\end{aligned}
$$

For the reflected beam in Figure 5-4,

$$
\left|\begin{array}{ll}
A & B \\
C & D
\end{array}\right|=\left|\begin{array}{ll}
1 & 0 \\
\frac{2 n_{1}}{R_{s}} & 1
\end{array}\right| \text {, }
$$

"A. Yario, "Quantum Electronics" 


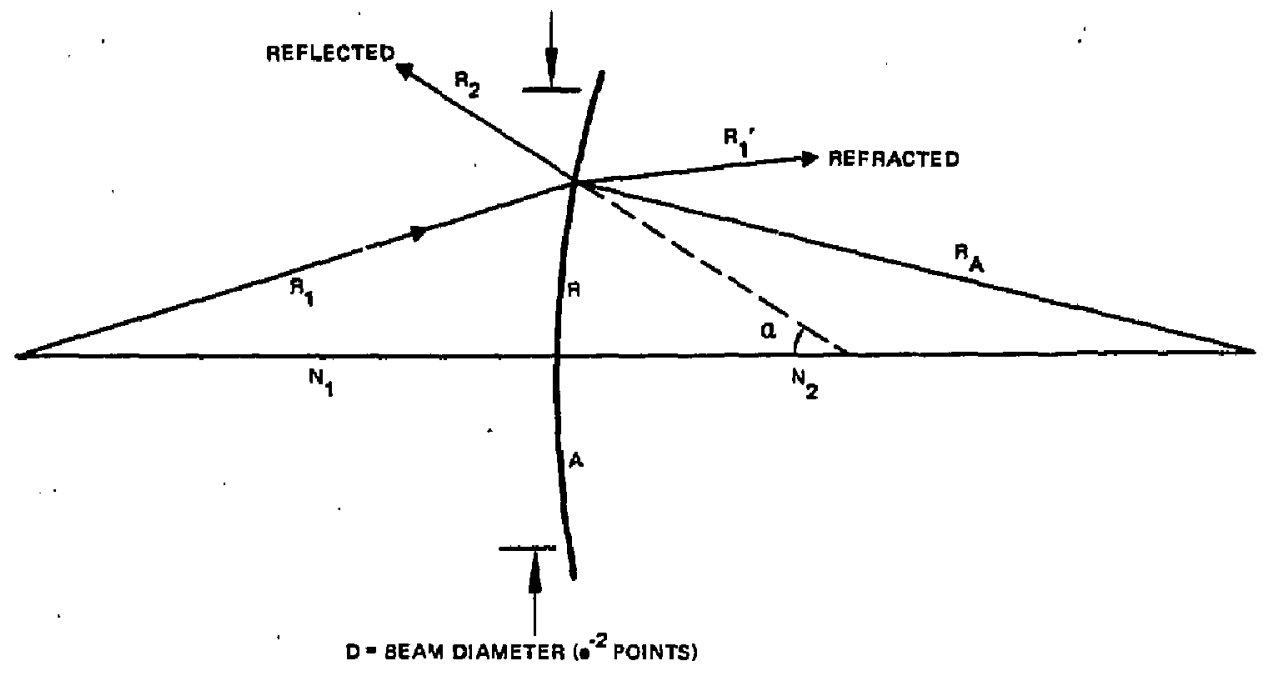

Figure 5-4, Geometry of a specular reflection.

and for the transmitted beam,

$$
\left|\begin{array}{ll}
A & B \\
C & D
\end{array}\right|=\left|\begin{array}{ll}
1 & 0 \\
\frac{-\left(n_{2}-n_{1}\right)}{R_{s}} & 1
\end{array}\right|
$$

The reflected curvature is

$$
\frac{-n_{1}}{R_{2}}=\operatorname{Re}\left(\frac{1}{q_{2}}\right)=\operatorname{Re}\left[\frac{\left(\frac{2 n_{1}}{R_{s}}\right) q_{1}+1}{q_{1}+0}\right]=\frac{2 n_{1}}{R_{s}}+\frac{n_{1}}{R_{1}},
$$

or

$$
-\frac{1}{R_{2}}=\frac{2}{R_{s}}+\frac{1}{R_{1}}
$$


and the refracted curvature is

$$
\frac{n_{2}}{R_{1}}=\frac{-\left(n_{2}-n_{1}\right)}{R_{S}}+\frac{n_{1}}{R_{1}}
$$

The conventions used here are that the index is set negative for propagation along the $-E$ axis, and $R_{s}$ is positive if centered to the right.

Equation (5-36) may be used to trace through a series of surfaces, at each of which (5-35) is used to calculate the reflected power that is intercepted by the receiver. The heterodyne signal may be written as in (5-30) with the integral evaluated at the surface $\mathrm{S}$ :

$$
\begin{aligned}
& E_{L O} \sim e^{-r^{2}}\left(\frac{1}{w^{2}}+\frac{i k_{0} n_{l}}{2 R_{1}}\right)_{\text {(LO field) }} \\
& -r^{2}\left(\frac{1}{w^{2}}+\frac{i k_{0} n_{1}}{2 R_{2}}\right) \quad(\text { reflected field }) \\
& \mathrm{E}_{\mathrm{R}} \sim \mathrm{e} \\
& I-\int \frac{\mathrm{d} r}{E_{L O}} E_{\mathrm{R}}^{*} \quad \text { (peak IF current) } \\
& -\int_{0}^{\infty} r d r e r^{2}\left[\frac{2}{w^{2}}+\frac{i k_{o} n_{1}}{2}\left(\frac{1}{R_{1}}-\frac{1}{R_{2}}\right)\right]
\end{aligned}
$$

The effective reflection coefficient is, for $p=$ specular reflection $=0.1$ percent for AR coatings, 


$$
\begin{aligned}
R & =\rho \frac{I\left(\frac{1}{\mathrm{R}_{1}}-\frac{1}{\mathrm{R}_{2}}\right)}{I(0)} \\
& =\frac{\rho}{1+\left[\frac{k_{0} w^{2} n_{1}}{4}\left(\frac{1}{R_{1}-R_{2}}\right)\right]^{2}} \\
R & =\frac{\rho}{1+\left(k_{0} w^{2} n_{1}\right)^{2}\left(\frac{1}{R_{s}}+\frac{1}{R_{1}}\right)^{2}}
\end{aligned}
$$

using Equation (5-35). The propagation of the curvature across the spaces between the surfaces will be approximated by

$$
R_{1}^{\prime \prime}=R_{1}{ }^{\prime}+L
$$

where

$$
\begin{aligned}
L & =\text { spacing to next surface } \\
R_{1}{ }^{\prime \prime} & =\text { radius of curvature at the new surface }
\end{aligned}
$$

This ray approximation should be adequate when the surfaces are far away from the focused beam waists.

Relations $5-36,-37$, and -38 are now used to fill out the entries in Table 5-1), with $p=0.001$. It is concluded that specular reflections are not a problem for the configurations analyzed.

If a surface is found to produce a substantial ghost, interference of the ghose with the target signal can be eliminated by adjusting their relative path lengths so that the peaks of the ghost response curve fall between those of the signal. Of course, the target signal is distinguishable from ghosts by its movement with path length adjustment. 
TABLE 5-1. GHOST REFLECTANCES

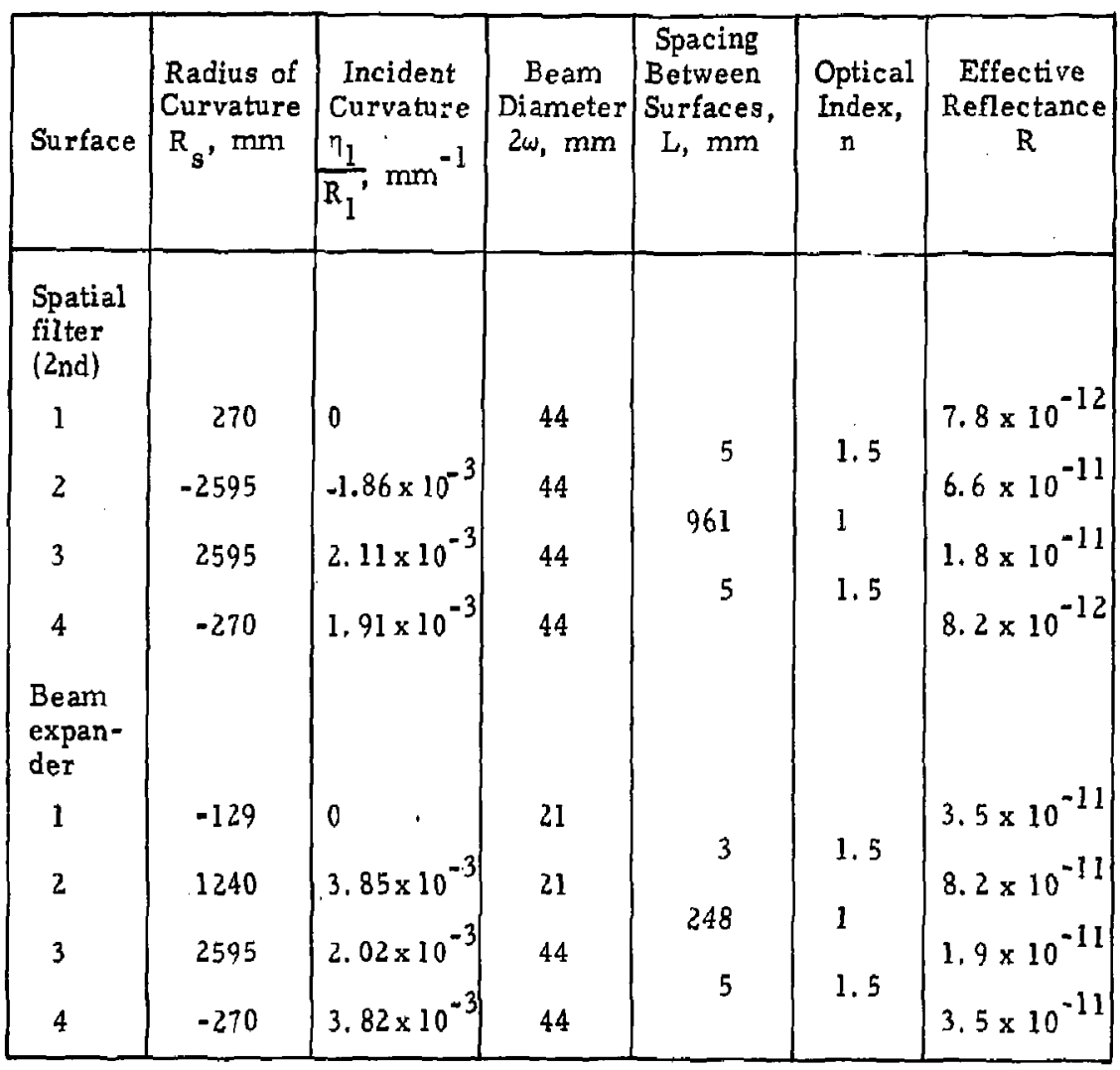

Finally, diffuse reflectance of surfaces will be considered. The effective reflectance for a Lambertian reflector may be written

$$
R^{\prime}=\rho^{\prime} \frac{\theta^{2}}{4}
$$

where

$\rho^{\prime}=$ surface reflectance into $2 \pi$ sterad.

$\theta=\frac{2 \lambda}{w}=\begin{aligned} & \text { diffraction limited divergence of a gaussian beam of } \\ & \text { radius } w \text {. }\end{aligned}$ 
$\pi \theta^{2} / 4$ is the solid angle intercepted by the receiver. It is seen that $R^{\prime}$ is largest for small $w$; therefore, letting

$$
\begin{aligned}
2 w & =21 \mathrm{~mm} \\
p^{\prime} & =5 \times 10^{-3},
\end{aligned}
$$

obtain

$$
R^{\prime}=4.6 \times 10^{-12}
$$

The behavior of $(5-39)$ with $w$ tells us that no trouble will derive from diffuse reflectance; if the system works at all with a 1 or $4 \mathrm{~mm}$ beam at the beamsplitter, then it will work the presence of the larger $w$ present in the amplifier chain. 


\subsection{SYSTEM PERFORMANCE ESTIMATE}

The performance is contained in Equation (5-29):

$$
\operatorname{SNR}_{m}(\delta X)=\left(\frac{\delta X}{\Delta}\right) F_{2}\left(\frac{\Delta}{2 \sigma}\right) \quad\left(\frac{\eta<P_{s}>T_{i}}{h v}\right)^{1 / 2}
$$

Substituting the values

$$
\begin{aligned}
& 2 \sigma=\Delta=\text { LO spacing }=6.44 \mathrm{~cm} \\
& F_{2}(1)=2.9 \text { (maximum) } \\
& \eta=\text { photodiode quantum efficiency } \approx 0.2 \text { : } \lambda=1.06 \mathrm{H} \\
& h v=2 \times 10^{-19} \text { joules } \\
& T_{i}=\text { phase lock amplifier integration tim }=1 \mathrm{sec} \\
& \left\langle P_{\vec{s}}\right\rangle=\text { signal power } \approx\left\langle\mathrm{P}_{\mathrm{L}}\right\rangle \text { (average last } \mathrm{r} \text { output power) } \\
& x \quad 1 / 4 \text { (attenuation from poiarizer in signal } \\
& \text { path) } \\
& \begin{array}{l}
\times 1 / 2 \text { (attenuatic fron chopper in signal } \\
\text { path) }
\end{array} \\
& \times 10^{-9} \text { (round trip attenuation in signal } \\
& \text { path for linea:ly polarized beam), }
\end{aligned}
$$

5-29 becomes, for a carrier to noise ratir, E.NR $R_{c} \gg 1$,

$$
S N R_{m}=503<\sum_{L}^{P}>^{1 / 2} \delta X . \quad(\delta X \text { in } \mathrm{mm})
$$

The above value of $S: R_{m}$ is val:d at all values of $S N R{ }_{c}$ if the carrier were synchronously demoilulated. However, the present system employs envelope detection whith degrades $S N R_{m}$ if $S N R_{c} \leq 1$. This effect may be incorporated into uquation $5-30$ by raultiplying by the faclor $Y_{1}$ which has been 
calculated for a linear rectifier in Appendix A. The results of Appendix A are summarized in the curve given in Figure 5-4 (a linear rectifier is equivalent to an envelope detector with short integration time, and probably provides a worst case estimate for $\gamma_{1}$ ), $\operatorname{SNR}_{c}$ is obtained from 5-9:

$$
\operatorname{SNR}_{c}(\tau)=\left(\frac{\eta<\dot{P}_{S}>}{h v B_{I F}}\right)^{1 / 2}\left(\frac{I_{s}(\tau)}{I_{s}(0)}\right) .
$$

where

$$
\begin{aligned}
B_{I F} & =100 \mathrm{kHz} \\
\frac{I_{s}(T)}{I_{s}(0)} & =e^{-1} \text { (the point at which SNR }{ }_{m} \text { is maximized). }
\end{aligned}
$$

Using the se values and those incorporated ir 5-30, we obtain

$$
\operatorname{SNR}_{c}=13 \cdot\left\langle\mathrm{P}_{\mathrm{L}}\right\rangle^{1 / 2}
$$

The iaser output when stabiy mode locked is $P_{L} \sim 0.5$ watts, leading to

$$
\operatorname{SNR}_{c}=9 .
$$

The obsierved SNR ${ }_{c}$ under these conditions is about 2.5, so that a degradation factor of $\gamma_{2} \approx 0.3 \mathrm{in} \mathrm{SNR}$ is experienced. It is seen from Figure 5-5 that $y_{1}=1$ under these conditions, and 5-30 becomes

$$
\mathrm{SNR}_{m}^{\prime}=(503) \gamma_{1} \gamma_{2} \gamma_{3}<\mathrm{P}_{L}>^{1 / 2} \delta \mathrm{X} \simeq 50 \delta \mathrm{X},
$$




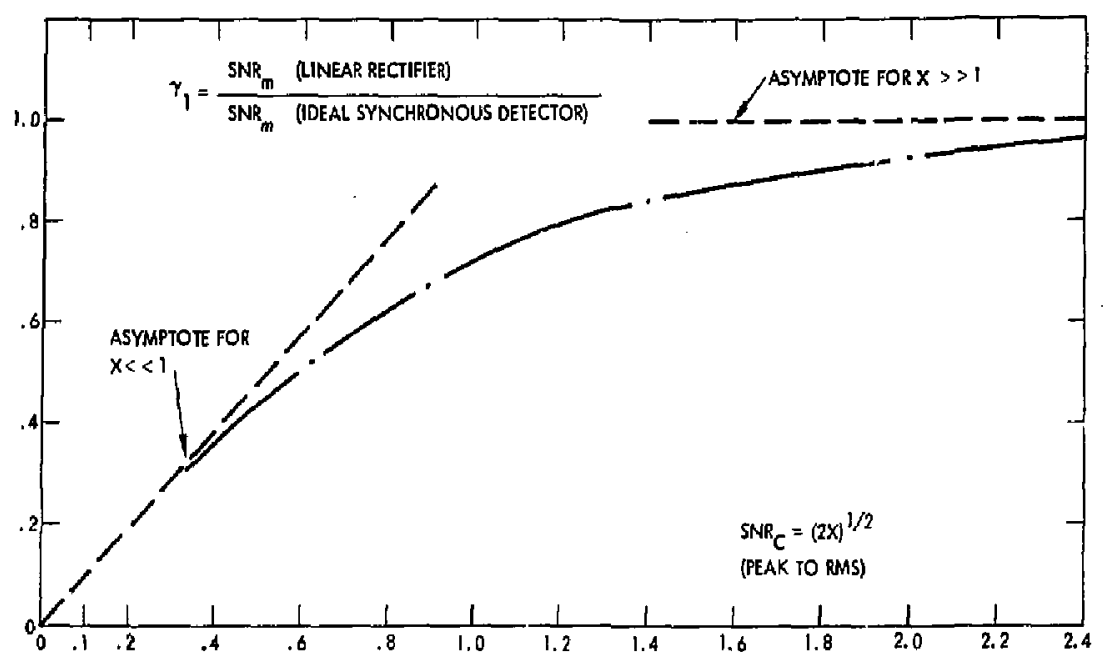

Figure 5-5. Degradation factor.

where $Y_{1} \approx 1, \gamma_{2} \approx 0.3$, and the additional degradation factor $\gamma_{3} \approx 0.5$ is assumed to account for the losses in carrier duty cycle. If the reasonable as sumption is made that $\mathrm{SNR}_{\mathrm{m}}=5$ is adequate to as sure a null, then 5-32 indicates a position accuracy

$$
|\delta X|<\frac{S N R m}{50}=0.1 \mathrm{~mm}
$$

for an attenuation of $10^{9}$. The positional error over a range of attenuations is plotted in Figure 5-6, from which it is seen that the loss incurred with envelope detection is significant only for transmissions less than $10^{-9}$. 


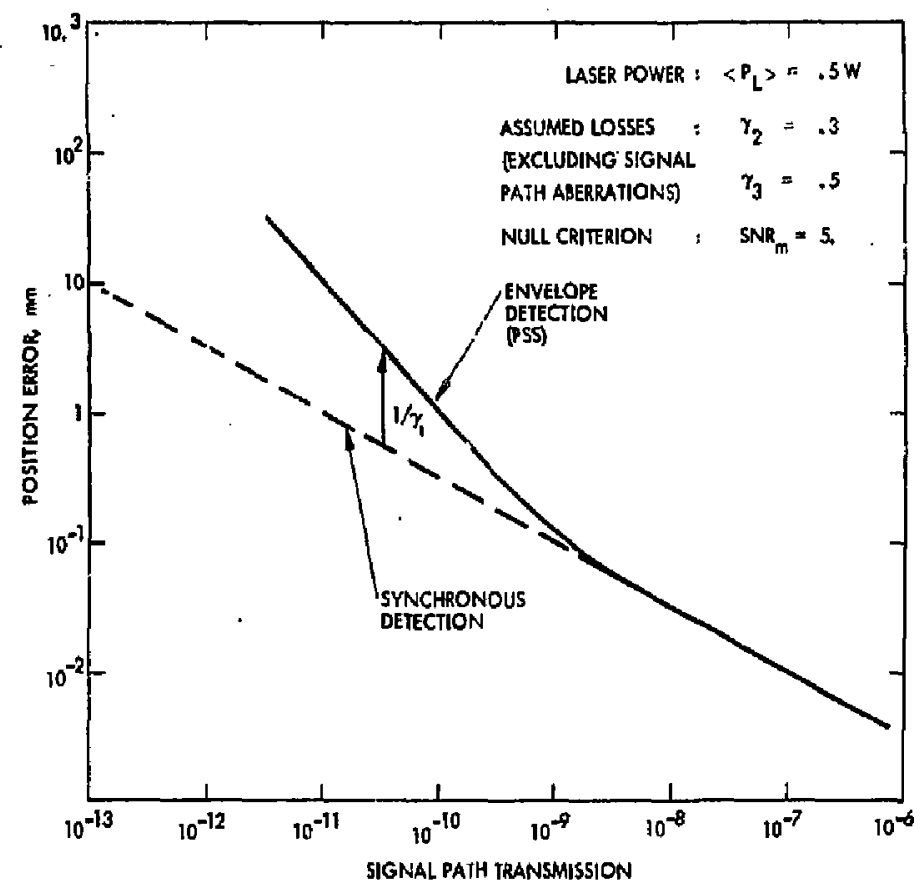

Figure 5-6. Estimated position error versus signal path transmission. 


\subsection{APPENDIX CALCULATION OF DEGRADATION IN SNR $\mathrm{m}$ DUE TO ENVELOPE DETECTION}

Synchronous detection of a modulated carrier involves mixing with the unmodulated carrier. A linear rectifier or an envelope detector works equally well with $A M$ modulation when the IF carrier signal-to-noise ratio $\left(\mathrm{SNR}_{c}\right)$ is greater than one. The rectifying diode recovers the correct phase of the carrier by attenuating the negative portion of the carrier cycle. When $\operatorname{SNR}_{c}$ falls below one, then this diode phase detection is often in error, and the final detection $\mathrm{SNR}_{\mathrm{m}}$ suffers by a factor $\gamma_{1} \leq 1 . \gamma_{1}$ is calculated below' for a linear rectifier (as compared to synchronous detection); this result is thought to be approximately correct for envelope detection as employed in the hardware.

Assume the initial (IF) waveforms:

$$
\begin{aligned}
& \text { Signal }=P \cos p t \\
& \text { Noise }=\eta_{i}=\eta_{c}(t) \cos p t+\eta_{s}(t) \sin p t
\end{aligned}
$$

where

$$
\overline{\eta_{i}^{2}}=\psi_{0}
$$

For synchronous detection (multiplication by $\cos p t$ ) the detector outputs are:

$$
\begin{aligned}
& I_{d c}=P \cos ^{2} p t=P / 2 \\
& \overline{\eta_{0}^{2}}=\left(\eta_{i} \cos p t\right)^{2}=\frac{1}{4} \overline{\eta_{c}^{2}}=\frac{1}{4} \overline{\eta_{i}^{2}}=\psi_{o} / 4,
\end{aligned}
$$

and

$$
\operatorname{SNR}_{c}=I_{d c} / \sqrt{\overline{\eta_{0}^{2}}}=P / \psi_{o}^{1 / 2}=(2 x)^{1 / 2} \text {. }
$$


where

$x \equiv P^{2} / 2 \psi_{0}$

For linear rectification with characteristic

$$
I=\left\{\begin{array}{ll}
0, & v<0 \\
a v, & v>0
\end{array}\right\},
$$

The outputs are*

$$
\begin{aligned}
I_{d c} & =\alpha\left(\frac{\psi_{0}}{2 \pi}\right)^{1 / 2} e^{-x / 2}\left[(1+x) I_{0}\left(\frac{x}{2}\right)+x I_{1}\left(\frac{x}{2}\right)\right] \\
\overline{\eta_{0}^{2}} & =\frac{2 \alpha^{2} \psi_{0}}{\pi^{2}}(x+1)-I_{d c}{ }^{2} \\
& =\alpha^{2}\left(\frac{\psi_{0}}{2 \pi}\right)\left\{\frac{4}{\pi}(x+1)-e^{-x}\left[(1+x) I_{0}\left(\frac{x}{2}\right)+x I_{1}\left(\frac{x}{2}\right)\right)\right\}
\end{aligned}
$$

where $I_{0}$ and $I_{1}$ are the modified Bessel functions. Limiting values of $I_{0}$ and $I_{1}$ lead to

*Rice, S. O. "Mathematical Analysis of Random Noire," Bell System Technical Journal, Vols 23, 24. Equations 4, 2-3, -6. 


$$
\begin{aligned}
\eta_{0}^{2}(x=0) & =\frac{\alpha^{2}}{\pi^{2}} \psi_{0}(2-\pi / 2) \\
I_{d c}(x>>1) & =\frac{\alpha P}{\pi}=-\frac{\alpha}{\pi}\left(2 \psi_{0}\right)^{1 / 2} x^{1 / 2} \\
\sqrt{\eta_{0}^{2}}(x>>1) & =\frac{\alpha}{\pi} \psi_{0}^{1 / 2} \\
S N R_{c} & =I_{d c} / \sqrt{\eta_{0}^{2}}=(2 x)^{1 / 2} \text { for } x>>1 .
\end{aligned}
$$

The detection problem at issue is that of an amplitude modulating square wave resulting in an input carrier amplitude that varies between $P$ and $P+\Delta P$. The SNR to an increment $\Delta P$ is

$$
\frac{\Delta P}{\sqrt{\eta^{2}}} \frac{d I_{d c}}{d P}
$$

and the ratio $\gamma_{1}$ is

$$
\begin{aligned}
\gamma_{1} & =\frac{\text { SNR for linear rectification }}{\text { SNR for synchronous detection }} \\
& \left.=\frac{\left(\frac{x}{2}\right)^{1 / 2} e^{-x / 2}\left[I_{0}\left(\frac{x}{2}\right)+I_{1}\left(\frac{x}{2}\right)\right]}{\left\{\frac{4}{\pi}(x+1)-e^{-x}\left[(1+x) I_{0}\left(\frac{x}{2}\right)+x I_{1}\left(\frac{x}{2}\right)\right]\right]^{-2}}\right]^{1 / 2} \\
& =\left\{\begin{array}{cc}
1.0 & x>1 \\
0.96(2 x)^{1 / 2} & x<<1
\end{array}\right\},
\end{aligned}
$$

where the derivatives and asymptotec expressions for $\mathrm{I}_{0}$ and $\mathrm{I}_{1}$ have been used. Figure 5-4 is a plot of Equation (A-3). The asymptotes in Equations (A-4) are useful in plotting the position error versus signal error transmission in Figure 5-6. 
?

"This report was prepared as an account of work sponsored by the United States Government. Neither the United States por the United States Energy Researeh \& Development Administration, nor any of their employees, nor any of their contractors, subcontractors, or their employees, makes any watranty, express or implied, or assumes any legal liability or responsibility for the accuracy. completeness or usefulness of any information, apparatus, product or process disclosed, or represents that its use would not infringe privately.owned rights." 